\title{
Phonon Boltzmann Transport Equation Based Modeling of Time Domain Thermo-Reflectance Experiments
}

\section{Syed Ashraf Ali and Sandip Mazumder ${ }^{+}$}

Department of Mechanical and Aerospace Engineering

The Ohio State University,

Columbus, $\mathrm{OH} 43210$, USA

Submitted to: International Journal of Heat and Mass Transfer

${ }^{+}$Author for correspondence

Dr. Sandip Mazumder

Department of Mechanical and Aerospace Engineering

The Ohio State University

Suite E410, Scott Laboratory

201 West $19^{\text {th }}$ Avenue

Columbus, $\mathrm{OH} 43210$

E-mail: mazumder.2@osu.edu

Phone: (614) 247-8099

Fax: (614) 292-3163 


\begin{abstract}
Time Domain Thermo-Reflectance (TDTR) experiments have been recently identified as a viable pathway toward extracting the phonon mean free path spectrum of semiconductor materials. However, this requires an intervening model. It is now widely believed that the frequency and polarization dependent phonon Boltzmann Transport Equation (BTE) is the most suitable model for this purpose. In this article, TDTR experiments are simulated using large-scale parallel computations of the phonon BTE in a two-dimensional computational domain. Silicon is used as the candidate substrate material. Simulations are performed for multiple pulse and modulation cycles of the TDTR pump laser. This requires resolution of a picosecond laser pulse within a computational timeframe that spans several hundreds of nanoseconds. The metallic transducer layer on top of the substrate is modeled using the Fourier law and coupled to the BTE within the silicon substrate. Studies are conducted for four different laser spot sizes and two different modulation frequencies. The BTE results are fitted to the Fourier law, and effective thermal conductivities are extracted. It is demonstrated that the time delay of the probe laser could have a significant effect on the fitted (extracted) thermal conductivity value. The modulation frequency is found to have negligible effect on the thermal conductivity, while the spot size variation exhibits significant impact. Both trends are found to be in agreement with experimental observations. The thermal conductivity accumulation function is also computed, and the effect of the mean free path spectrum on the thermal conductivity suppression is delineated.
\end{abstract}

Keywords: Boltzmann Transport Equation; phonon; heat transfer; Time Domain Thermo-Reflectance; TDTR; thermal conductivity 


\section{INTRODUCTION}

Heat conduction in semiconductor materials primarily occurs by vibrational waves traveling through the lattice. From a quantum mechanical standpoint, these waves may be thought of as discrete energy packets, called phonons [1-3]. The mean free path of phonons in silicon at room temperature is approximately in the range $10-10^{5} \mathrm{~nm}$, with the average around $300 \mathrm{~nm} \mathrm{[3],} \mathrm{which} \mathrm{could} \mathrm{be} \mathrm{significantly} \mathrm{larger} \mathrm{than} \mathrm{the} \mathrm{characteristic}$ feature sizes in modern-day applications of semiconductor materials. If the mean free path of the phonons is larger than or of the same order of magnitude as the characteristic length scale, thermodynamic equilibrium ceases to exist, and the Fourier law of heat conduction, which assumes local equilibrium, becomes invalid. The Boltzmann Transport Equation (BTE) for phonons serves as a viable alternative $[1,2,4]$ for the prediction of non-equilibrium heat conduction in semiconductor materials at the nanoscale.

One of the critical inputs to the phonon BTE is the phonon relaxation time-scale. In a pure semiconductor, since phonons relax to equilibrium primarily due to phononphonon interactions or intrinsic scattering, the scattering time-scale may be deemed the most important input to the BTE. Another important input is the wavevector-frequency or dispersion relationship. The slope of the dispersion curve provides the group velocity of the phonons. The mean free path of a phonon is the product of its group velocity and its scattering time-scale. Since the group velocity and scattering time-scale are both frequency dependent, phonons of different frequency have different mean free paths. The frequency dependent mean free path is often referred to as the mean free path spectrum. In a nanoscale system, not all phonons contribute equally to thermal transport. Phonons 
with large mean free paths (ballistic phonons) interact more frequently with geometric boundaries than with other phonons, while phonons with small mean free paths (diffusive phonons) engage in frequent inter-phonon scattering. Therefore, from an engineering standpoint, it is conceivable that if the mean free path spectrum of phonons in a material was known, one could tailor the geometric structure in a manner that would either enhance or suppress the thermal conductivity of the nanostructure by allowing or disallowing phonons of certain frequencies to participate in thermal transport. Hence, determination of the phonon mean free path spectrum in semiconductor materials is currently considered a translational area of research within the nanoscale heat transfer community [5].

Of the aforementioned two quantities that constitute the mean free path, namely the dispersion relationship and the scattering time-scale, the scattering time-scale is the more challenging to determine. Computation of the scattering time-scale of various phonon modes and frequencies is an active area of research. Starting from the works of Callaway [5] and Holland [6,7] in the 1960s, various approaches have been used to estimate three-phonon scattering rates. These include perturbation techniques pioneered by Klemens [8] in combination with the selection rules of collision, molecular dynamics [10-14], collision integral methods [15-18], ab initio methods combined with Fermi's golden rule [19-24], among others. As to which method is most appropriate for the task at hand is a highly controversial subject. Worth noting is the variability in the values of the scattering time-scales predicted by various methods $[8,9,14,18,20]$. Despite significant variability in the predicted scattering time-scales, researchers in this area typically demonstrate "good" agreement between their predicted effective thermal conductivity 
and some selected experimental data. Although the common notion is that the scattering time-scales predicted by first-principles methods [19-24] are more accurate than the timescales proposed by earlier works [8,9], there is no direct evidence in the form of a comparative study to support this notion. More importantly, thermal conductivity is the net manifestation of the propagation of phonons of many different modes and frequencies and their interactions with geometric boundaries, and agreement (model versus experiment) in effective thermal conductivity neither validates nor disproves the predicted scattering time-scales. What is needed is direct validation of the mean free path spectrum against experimental measurements, or conversely, its direct extraction from experimental data, i.e., without taking the thermal conductivity route.

Experiments at the nanoscale are limiting in scope. Until recently, experiments have only been able to provide effective thermal conductivity of thin semiconductor films as a function of film thickness, temperature, and dopant concentrations [4]. While it is possible now to measure phonon relaxation times directly using inelastic neutron scattering, x-ray scattering, or Brillouin scattering [25,26], the data obtained by such methods do not span the entire frequency range of the phonons that are believed to be the carriers of heat $[4,27]$. In the past decade, a new class of experiments-so-called laser based pump probe experiments - hold the promise of divulging significantly more information than just the effective thermal conductivity of the material under scrutiny [27-33]. Experimental techniques that belong to this category are transient thermal grating, time domain thermo-reflectance (TDTR), frequency domain thermo-reflectance, and their variations. An excellent recent review of these techniques is provided by Regner et al. [5]. Although the details are somewhat different in these experiments, they all share 
the common philosophy of heating a designated spot on a substrate with one laser beam (the pump laser) and monitoring temperature evolution with another one (the probe laser). The temperature response of the hot spot is finally analyzed (fitted to a model) to extract the thermal conductivity. Furthermore, in approaches based on thermoreflectance, the detection principle requires the power of the heating laser to be modulated, and in the case of TDTR, a pulse train is implemented, thereby enabling — at least, in principle - identification of the dominant phonon frequencies that contribute to thermal transport and the effective thermal conductivity.

It is now unanimously acknowledged [5] that the BTE for phonons is capable of describing non-equilibrium thermal transport in semiconductors, and is an appropriate intervening model for describing the causal relationship between the spectral scattering time-scale of phonons, the geometry in question, and the resulting temperature and heat flux distributions. The determination of the spectral scattering time-scale from measured temperature, of course, constitutes an inverse problem. Nonetheless, prior to solution of this inverse problem, it is first necessary to conduct forward calculations that will predict temperature and heat flux with the spectral scattering time-scale as inputs. This implies that if TDTR is the experimental technique being used, one must first be able to simulate TDTR experiments exactly using the modeling framework, namely the multi-dimensional frequency-dependent phonon BTE. This is a critical barrier that must first be overcome [5] prior to any inverse analysis and determination of the spectral scattering time-scale directly from experimental measurements.

The solution of the phonon BTE is rendered computationally challenging by the fact that even under the single time relaxation approximation, it is a seven-dimensional 
equation. One method that has found prolific use in the solution of the phonon BTE is the Monte Carlo method. The method was first adapted and applied to the frequency and polarization dependent phonon BTE by Mazumder and Majumdar [34] in 2001, and since its inception, has been used by many other researchers $[35,36]$. One of the shortcomings of the Monte Carlo method is the presence of inherent statistical error in the solution. In recent years, the variance reduction Monte Carlo method has been adapted [37] to significantly reduce statistical noise in phonon BTE computations. Indeed, this enhancement has enabled its use in the simulation of scenarios commonly encountered in TDTR experiments $[37,38]$. The other approach that has found prolific use in the solution of the phonon BTE is discretization (in physical space, angular space, and frequency space) based deterministic methods. Such methods were brought to the limelight by Murthy and co-workers [39-42]. To bring the state-of-the-art in deterministic solution of the phonon BTE into perspective, until 2010, the best example of multi-dimensional computation of the frequency-dependent BTE was the one by Ni and Murthy [43] who performed calculations on a 2D rectangular domain with $80 \times 80$ cells, 64 angles, and 80 spectral bands, resulting in $\sim 3 \times 10^{7}$ unknowns. In the past five years, however, significant progress has been made in the solution of the phonon BTE using deterministic techniques [44-47]. In particular, Ali et al. [46] demonstrated a parallel BTE solver for unsteady solution of the phonon BTE with the inclusion of dispersion and polarization in threedimensional geometries discretized using 40 spectral bands, 400 angles, and about 0.7 million cells (spatial nodes), resulting in approximately 10 billion unknowns.

Despite significant progress in the solution of the phonon BTE, simulation of TDTR experiments using the phonon BTE remains elusive. This is because in TDTR 
experiments the pump laser pulse is usually of $\sim 1$ picosecond duration, while the experimental data is gathered over a period of several hundred nanoseconds [32,33]. In addition to the picosecond pulses, the laser power is also modulated over a time scale spanning several tens of nanoseconds. The multiple laser pulses create an accumulation effect [32] that cannot be captured by simulating a single laser pulse. Resolution of these disparate time scales makes multi-dimensional BTE calculations computationally challenging. Despite this challenge, in realization of the fact that the BTE encapsulates the relevant phonon physics, attempts have been made to simulate pump probe experiments by using the one-dimensional (1D) BTE. Regner et al. [48] solved the 1D BTE in frequency domain in the cross-plane direction and explained the so-called ballistic resistance and the effect of the pump laser's modulation frequency on the thermal penetration depth. Both Regner et al. [48] and Minnich and co-workers $[38,49,50]$ demonstrated extraction of the so-called thermal conductivity accumulation and suppression functions from 1D BTE calculations. One limitation of such studies is that they do not consider the interplay of multiple length scales, viz. the laser spot size and the penetration depth (which depends on the laser modulation frequency). This can only be accomplished through solution of the multi-dimensional BTE. The only known attempt at using the solution to the multi-dimensional BTE in time domain for TDTR experiments is a Monte Carlo study [38] that simulated only a single power pulse of the pump laser. Although preliminary, this study indicates that phonon transport rates and the ensuing suppression function is different in the cross-plane and in-plane directions, and the existence of multiple length scales complicates the phonon transport process, resulting in different thermal conductivity suppression in the different directions. 
The work presented in this article represents the first attempt at simulating the conditions of a typical TDTR experiment using a spatially two-dimensional (2D) solution to the frequency and polarization dependent phonon BTE in time domain. 2D planar computations are computationally significantly cheaper than $2 \mathrm{D}$ axisymmetric calculations because of the number of angular BTEs that need to be solved (see later), and are, thus, considered in this preliminary study to keep computational times tractable. Unsteady calculations are conducted over a total time period covering two full modulation cycles of the pump laser. Silicon is used as the candidate substrate material. The exact same conditions as used in the BTE computations are also simulated using a Fourier model, and the data are processed to extract the effective thermal conductivity, heat flux at various locations along the substrate, and thermal conductivity accumulation functions.

\section{THEORY AND MATHEMATICAL FORMULATION}

\subsection{Boltzmann Transport Equation}

The BTE for phonons, under the single time relaxation approximation, may be written as [1]

$\frac{\partial f}{\partial t}+\mathbf{v} \nabla f=\frac{f_{0}-f}{\tau}$

where $f$ is the distribution function of an ensemble of phonons, $f_{0}$ is the equilibrium number density function (i.e., the Bose-Einstein distribution function), $\tau$ is the overall relaxation time-scale of the phonons to relax to equilibrium due to all scattering processes in combination, and $\mathbf{v}$ is the group velocity. For an isotropic wavevector space, the 
distribution function, $f$, is a function of 8 independent variables, i.e., $f=f(t, \mathbf{r}, \hat{\mathbf{s}}, \omega, p)$, where $t$ is time, $p$ is polarization, and $\omega$ is the angular frequency. The space vector $\mathbf{r}$ has 3 components ( $x, y$, and $z$ in Cartesian coordinates), while the direction vector $\hat{\mathbf{s}}$ has 2 components, namely the polar angle $\theta$, and the azimuthal angle $\psi$. Since polarizations are discrete, it is customary to think of the distribution function as being a function of seven independent variables, with the implicit understanding that it is different for different polarizations. The Bose-Einstein distribution, $f_{0}$, on the other hand, is independent of direction and polarization, i.e., $f_{0}=f_{0}(t, \mathbf{r}, \omega)$. The group velocity, $\mathbf{v}$, is a function of direction, angular frequency, and polarization, i.e., $\mathbf{v}=\mathbf{v}(\hat{\mathbf{s}}, \omega, p)$, while the relaxation (or overall scattering) time-scale, $\tau$, is a function of angular frequency, temperature, and polarization i.e., $\tau=\tau(\omega, T, p)$. Equation (1) can be written in terms of the phonon intensity as follows $[1,51]$ :

$\frac{\partial I_{\omega, p}}{\partial t}+\mathbf{v}_{\omega, p} \nabla \nabla I_{\omega, p}=\frac{\partial I_{\omega, p}}{\partial t}+\left|\mathbf{v}_{\omega, p}\right| \nabla \square\left(I_{\omega, p} \hat{\mathbf{s}}\right)=\frac{I_{0, \omega, p}-I_{\omega, p}}{\tau_{\omega, p}}$,

where $\mathbf{v}_{\omega, p}=\left|\boldsymbol{v}_{\omega, p}\right| \hat{\mathbf{s}}$, and the phonon intensities are defined as [51]

$$
\begin{aligned}
& I_{\omega, p}=I(t, \mathbf{r}, \hat{\mathbf{s}}, \omega, p)=\left|\mathbf{v}_{\omega, p}\right| \hbar \omega f D(\omega, p) / 4 \pi \\
& I_{0, \omega, p}=I_{0}(t, \mathbf{r}, \omega, p)=\left|\mathbf{v}_{\omega, p}\right| \hbar \omega f_{0} D(\omega, p) / 4 \pi
\end{aligned},
$$

where $I_{\omega, p}$ is the spectral directional phonon intensity, while $I_{0, \omega, p}$ is the equilibrium spectral phonon intensity. $D(\omega, p)$ is the phonon density of states per unit volume, and $\hbar$ is the Dirac constant, or reduced Planck constant. In order to emphasize that in the frequency dependent BTE, the phonon intensity, relaxation time-scale and group velocity are functions of both frequency and polarization, the subscripts $\omega$ and $p$ have been 
introduced. In addition, since the phonon intensity is a function of direction, frequency, and polarization, Eq. (2) must be solved for all directions and frequencies, and summed over all directions, frequencies, and polarizations in order to compute the heat flux at any spatial location and instant of time, as is discussed next.

\subsection{Heat Flux, Divergence of Heat Flux, and Temperature}

The heat flux is related to the phonon intensity by the relationship [1,51]

$$
\mathbf{q}(t, \mathbf{r})=\sum_{p} \int_{\omega_{\min , p}}^{\omega_{\max , p}} \int_{4 \pi} I_{\omega, p}(t, \mathbf{r}, \hat{\mathbf{s}}, \omega, p) \hat{\mathbf{s}} d \Omega d \omega=\sum_{p} \int_{\omega_{\min , p}}^{\omega_{\max , p}} \mathbf{q}_{\omega, p}(t, \mathbf{r}) d \omega
$$

where the integrals are over all solid angles $(\Omega)$ and the frequency $(\omega)$ range of each polarization. $\omega_{\max , p}$ and $\omega_{\min , p}$ are the maximum and minimum frequencies, respectively, corresponding to a given polarization, $p$. In Eq. (4), $\mathbf{q}_{\omega, p}$ denotes the spectral heat flux while $\mathbf{q}$ denotes the total heat flux at any instant of time, $t$, and location whose position vector is denoted by $\mathbf{r}$. Once the BTE [Eq. (2)] has been solved for all directions and frequencies, Eq. (4) must be used to compute the heat flux. While the solution of the BTE provides a mechanism to determine the heat flux, in order to determine the temperature distribution, one must apply the first law of thermodynamics. For a static medium, the first law may be written as [52]

$$
\frac{\partial u}{\partial t}=-\nabla \llbracket \mathbf{q}+\dot{q}_{g e n},
$$

where $u$ is the internal energy per unit volume and $\dot{q}_{g e n}$ is the heat generation rate per unit volume due to other mechanisms, such as electron-phonon or photon-phonon interactions. The right hand side of Eq. (5) may be reduced further by making use of Eq. 
(4) and the BTE [Eq. (2)]. To do so, we first integrate Eq. (2) over the total solid angle $4 \pi$ to yield

$$
\frac{\partial}{\partial t}\left(\int_{4 \pi} I_{\omega, p} d \Omega\right)+\left|\mathbf{v}_{\omega, p}\right| \nabla\left(\int_{4 \pi} I_{\omega, p} \hat{\mathbf{s}} d \Omega\right)=\frac{1}{\tau_{\omega, p}}\left(4 \pi I_{0, \omega, p}-\int_{4 \pi} I_{\omega, p} d \Omega\right)
$$

Using the definition of the directionally integrated spectral intensity,

$$
G_{\omega, p}=\int_{4 \pi} I_{\omega, p} d \Omega
$$

and the spectral heat flux given by Eq. (4), Eq. (6) may be re-written as

$$
\frac{\partial G_{\omega, p}}{\partial t}+\left|\mathbf{v}_{\omega, p}\right| \nabla \sqsubset \mathbf{q}_{\omega, p}=\frac{1}{\tau_{\omega, p}}\left(4 \pi I_{0, \omega, p}-G_{\omega, p}\right)
$$

Substitution of Eq. (8) into Eq. (5) yields

$$
\frac{\partial u}{\partial t}=-\sum_{p} \int_{\omega_{\min , p}}^{\omega_{\max , p}} \frac{1}{\left|\mathbf{v}_{\omega, p}\right|}\left[\frac{1}{\tau_{\omega, p}}\left(4 \pi I_{0, \omega, p}-G_{\omega, p}\right)-\frac{\partial G_{\omega, p}}{\partial t}\right] d \omega+\dot{q}_{g e n}
$$

The internal energy of a crystalline material at a given spatial location, $\mathbf{r}$, at equilibrium, is related to the local temperature through the Bose-Einstein distribution and its density of state [1]

$$
u(\mathbf{r})=\sum_{p} \int_{\omega_{\min , p}}^{\omega_{\text {max }, p}} u_{\omega, p} d \omega=\sum_{p} \int_{\omega_{\min , p}}^{\omega_{\text {max }, p}} f_{0} \hbar \omega D(\omega, p) d \omega=\sum_{p} \int_{\omega_{\min , p}}^{\omega_{\text {max }, p}} \frac{\hbar \omega D(\omega, p)}{\exp \left[\hbar \omega / k_{B} T(\mathbf{r})\right]-1} d \omega
$$

where the summation is over all polarization branches, and $k_{B}$ is the Boltzmann constant. $u_{\omega, p}$ denotes the contribution of phonons of frequency, $\omega$, and polarization, $p$, to the internal energy. Substitution of Eq. (10) into Eq. (9) yields a nonlinear equation in temperature, and the determination of the temperature field requires solution of this nonlinear equation. The temperature determined by this methodology is the local absolute temperature if local thermodynamic equilibrium exists. If the local number density 
distribution $(f)$ deviates from the equilibrium Bose-Einstein distribution $\left(f_{0}\right)$, the temperature computed using the above procedure is the so-called pseudo-temperature $[1,34]$, an may be interpreted as just a measure of the internal energy.

\subsection{Boundary Conditions to the BTE}

For the BTE [Eq. (2)], boundary conditions are necessary for the intensity. Two types of boundary conditions are generally used: (1) thermalizing, and (2) reflective.

A thermalizing boundary is analogous to a black surface for thermal radiation. It emits phonons based on the equilibrium energy distribution and absorbs any phonons that strike it, and is mathematically written as

$$
I_{\omega, p}\left(t, \mathbf{r}_{w}, \hat{\mathbf{s}}_{o}, \omega, p\right)=I_{0, \omega, p}\left(t, \mathbf{r}_{w}, \omega, p\right)
$$

where $\mathbf{r}_{w}$ is the position vector of the boundary or wall, and $\hat{\mathbf{s}}_{o}$ is the outgoing direction.

A reflective boundary condition is applied to adiabatic boundaries or symmetry planes. At such boundaries, the net normal heat flux is zero, which implies that the phonons are reflected without any loss of energy. The reflection of phonons can be diffuse, specular or partially specular. At a diffuse boundary, the incoming phonon intensity is split equally into all outgoing directions such that the net energy is conserved. Mathematically, this may be written as [53]

$$
I_{\omega, p}\left(t, \mathbf{r}_{w}, \hat{\mathbf{s}}_{o}, \omega\right)=\frac{1}{\pi} \int_{\hat{\mathbf{s}}_{i}\langle\hat{\mathbf{n}}<0} I_{\omega, p}\left(t, \mathbf{r}_{w}, \hat{\mathbf{s}}_{i}, \omega\right) \hat{\mathbf{s}} i \hat{\mathbf{n}} d \Omega_{i}
$$

where $\hat{\mathbf{s}}_{o}$ and $\hat{\mathbf{s}}_{i}$ are the outgoing and incoming directions, respectively. At a specular boundary, on the other hand, the incoming phonon intensity is diverted to the specular direction, which is given by [53] 
$\hat{\mathbf{s}}_{o}=\hat{\mathbf{s}}_{s}=\hat{\mathbf{s}}_{i}-2\left|\hat{\mathbf{s}}_{i}\right| \hat{\mathbf{n}} \mid \hat{\mathbf{n}}$

where $\hat{\mathbf{s}}_{s}$ is the direction of specular reflection, and $\hat{\mathbf{n}}$ is the surface normal pointing outward from the boundary. For partially specular surfaces, a combination of the above two formulations is warranted, yielding

$$
I_{\omega, p}\left(t, \mathbf{r}_{w}, \hat{\mathbf{s}}_{o}, \omega\right)=\alpha I_{\omega, p}\left(t, \mathbf{r}_{w}, \hat{\mathbf{s}}_{s}, \omega\right)+\frac{1-\alpha}{\pi} \int_{\hat{s}_{i} \hat{\mathbf{n}}<0} I_{\omega, p}\left(t, \mathbf{r}_{w}, \hat{\mathbf{s}}_{i}, \omega\right) \hat{\mathbf{s}}_{i}\left[\hat{\mathbf{n}} d \Omega_{i}\right.
$$

where $\alpha$ is the so-called degree of specularity [34,51], and represents the fraction of the incoming energy that is reflected back in the specular direction.

\subsection{Transducer-Substrate Interface Model}

In a typical TDTR experiment, the semiconductor substrate is covered with a metallic socalled transducer film, as shown schematically in Fig. 1. The transducer film is necessary to prevent generation of excessive free electrons within the semiconductor substrate (so that energy transport in the substrate is mainly due to phonons and not electrons), which is likely to occur if the laser were to strike the semiconductor substrate directly $[5,32]$. Rather, the laser energy directly excites the free electrons within the transducer film, which in turn, are thought to couple with the phonons within the semiconductor substrate. It has been observed that depending on the transducer material (aluminum is typically used in TDTR experiments), the measured thermal response of the substrate is different. It has been hypothesized [5] that the change in the response is due to change in the degree of electron-phonon coupling between the metallic transducer and the semiconductor substrate. A recent study [54] has indicated that the spectral transmission coefficient does impact the temporal response in a TDTR experiment. In this study, we employ a 
simplistic model in which heat conduction in the transducer is modeled using the Fourier law (since electron mean free paths are very small), while heat conduction in the semiconductor substrate is modeled using the phonon BTE. For coupling, it is assumed that electron energy is completely converted to phonon energy at the interface, and that the phonon number density function at the top surface of the substrate is the equilibrium distribution, i.e., the top surface of the substrate is thermalizing. Furthermore, since the transducer film is extremely thin, the thermal resistance across may be neglected, and it may be assumed to be a lumped mass (the Biot number was estimated to be approximately 0.04 for typical TDTR conditions). Under the aforestated assumptions, energy balance in the transducer film, following Fig. 2, yields

$$
L_{t} A \rho_{t} c_{p, t} \frac{d T}{d t}=\dot{Q}_{i n}-\dot{Q}_{\text {out }}=q_{L} A-\frac{T-T_{\text {top }}}{R_{C}}
$$

where $L_{t}$ is the thickness of the transducer film, and $A$ its top (or bottom) surface area. The density and specific heat capacity of the transducer material are denoted by $\rho_{t}$ and $c_{p, t}$, respectively. The pump laser power (flux) is denoted by $q_{L}(t)$, which, as indicated, is a function of time. The loss of heat from the transducer film occurs via conduction to the substrate underneath it. The temperature of the top surface of the substrate is denoted by $T_{\text {top }}$, as also shown in Fig. 2 . The contact resistance between the metal transducer and the semiconductor substrate is denoted by $R_{C}$. Equation (15) can be integrated to yield the average temperature, $T$, of the transducer at any instant of time, provided $q_{L}(t)$ and $\dot{Q}_{\text {out }}$ are known. While $q_{L}(t)$ is a known input quantity, $\dot{Q}_{\text {out }}$ is not. It is an output of the BTE calculation. Thus, coupling of Eq. (15) with the BTE [Eq. (2)] in the substrate 
requires a self-consistent iterative procedure, the discussion of which is deferred until the numerical solution to the BTE has been discussed.

\section{NUMERICAL PROCEDURE}

\subsection{Discretization of the BTE}

The BTE, namely Eq. (2), is spatially discretized using the unstructured finite-volume procedure [55]. For angular discretization, the CADOM [56,57] is used because it is known to mitigate so-called ray effects [56-58]. In the CADOM, the total solid angle, $4 \pi$, is discretized using pre-determined finite solid angles (or control angles), each centered along direction $\hat{\mathbf{s}}_{i}$, defined by [56] $\hat{\mathbf{s}}_{i}=\sin \theta_{i} \sin \psi_{i} \hat{\mathbf{i}}+\sin \theta_{i} \cos \psi_{i} \hat{\mathbf{j}}+\cos \theta_{i} \hat{\mathbf{k}}$, where $\theta_{i}$ and $\psi_{i}$ are polar and azimuthal angles, respectively, that define the discrete direction $i$. The BTE, after spatial, spectral, and angular discretization, may be written as [46]:

$$
\frac{\partial I_{i, j, p, k}}{\partial t} V_{k} \Omega_{i}+|\boldsymbol{v}|_{j, p, k} \sum_{f} I_{i, j, p, f} \mathbf{S}_{i}\left[\hat{\mathbf{n}}_{f} A_{f}=\frac{1}{\tau_{j, p, k}}\left(I_{0, j, p, k}-I_{i, j, p, k}\right) V_{k} \Omega_{i},\right.
$$

where the indices span the following values: $i=1,2, \ldots, N_{d i r}, j=1,2, \ldots, N_{b a n d}$, $k=1,2, \ldots, N_{c e l l}$, and $p \in \mathrm{LA}, \mathrm{TA}, \mathrm{LO}, \mathrm{TO} . I_{i, j, p, k}$ denotes the phonon intensity along the $i$-th direction (or solid angle), $j$-th spectral interval (or band), $p$-th polarization, and $k$-th cell (or control volume). The total number of directions (discrete solid angles) is denoted by $N_{d i r}$, the total number of bands by $N_{\text {band }}$, and the total number of cells by $N_{c e l l}$. $V_{k}$ denotes the volume of the $k$-th cell. $\Omega_{i}$ denotes the solid angle corresponding to the $i$-th direction, and is defined as

$$
\Omega_{i}=\int_{\Delta \theta_{i}} \int_{\Delta \psi_{i}} \sin \theta d \theta d \psi=2 \sin \theta_{i} \sin \left(\frac{\Delta \theta_{i}}{2}\right) \Delta \psi_{i}
$$


The equilibrium intensity of the $j$-th band, $p$-th polarization and $k$-th cell is denoted by $I_{0, j, p, k}$. The quantity, $\mathbf{S}_{i}$, in Eq. (16) is defined as follows:

$\mathbf{S}_{i}=\int_{\Delta \theta_{i}} \int_{\Delta \psi_{i}} \hat{\mathbf{s}}_{i} \sin \theta d \theta d \psi$

The intensity at the face, denoted by $I_{i, j, p, f}$, is an unknown in Eq. (16), along with the cell-center intensity, $I_{i, j, p, k}$. The intensity at the face needs to be expressed in terms of the cell-center values prior to solution of Eq. (16). The face intensity is generally approximated as the upwind cell's intensity $[39,40]$, i.e.,

$I_{i, j, p, f}= \begin{cases}I_{i, j, p, k} & \text { if } \quad \hat{\mathbf{s}}_{i}\left[\hat{\mathbf{n}}_{f}>0\right. \\ I_{i, j, p, N} & \text { if } \quad \hat{\mathbf{s}}_{i}\left[\hat{\mathbf{n}}_{f}<0\right.\end{cases}$

where $N$ denotes the neighbor to the $k$-th cell, and $\hat{\mathbf{n}}_{f}$ is the outward-pointing surface normal to face $f$ from the perspective of cell $k$, as shown in Fig. 3.

\subsection{Overall Solution Procedure}

Determination of the temperature and heat flux distributions in a simulation of a typical TDTR experimental setup requires solution of the phonon BTE [Eq. (16)] in conjunction with the first law [Eq. (9)]. In this study, a segregated solution approach is used, in which the aforementioned two equations are solved sequentially and coupled using an outer loop iteration within each time step, as depicted in the overall solution algorithm shown in Fig. 4. After spectral (band-wise) and angular (direction-wise) discretization, the solution of the BTE essentially reduces to the solution of $N_{b a n d} \times N_{d i r}$ three-dimensional (time and two space variables) partial differential equations. These partial differential equations, pending spatial and temporal discretization, may be solved in parallel. 
Previous research [46] has explored both band-wise and direction-wise parallelization, and it was found that band-wise pararrelization is the most efficient because the communication overheads are the lowest and the computational load is best balanced. Therefore, in this study, band-wise parallelization is employed. A discussion of the parallel algorithms is beyond the scope of this article, and the reader is referred to Ref. [46] for additional details. In the present study, the linear systems arising out of finite volume discretization of the individual spatio-temporal BTEs are solved using the Generalized Minimal Residual (GMRES) solver [59] after incomplete LU (ILU) preconditioning. 20 Krylov sub-spaces are used, and 6 orders of magnitude reduction of residual are enforced for the GMRES solver. Time advancement is implemented using the backward Euler procedure [55], which has the advantage of being unconditionally stable for any time step size. A variable time-step is used, in which the time-step is increased using a geometric progression within each power cycle, i.e., $(\Delta t)_{n}=n\left[(\Delta t)_{n-1}\right]$. This implies that at the first time-step, during which the pump laser is on, a time-step size of $(\Delta t)_{0}$ is used. In subsequent time-steps, when the pump laser is off, progressively larger time-step sizes are used, until the full on-off cycle concludes, and it is time for a new laser pulse.

One important step in the simulation of TDTR experiments is the determination of the temperature at the transducer-substrate interface, as also highlighted in the overall solution algorithm shown in Fig. 4. The physical model and underlying assumptions to accomplish this task have already been discussed in Section 2.4. In the numerical implementation of Eq. (15), since the pump laser flux, $q_{L}(t)$, is constant $\left(=q_{0}\right)$ during a time step, Eq. (15) can be integrated to obtain a closed-form analytical solution written as 
$T\left(t_{n+1}\right)=\left[T\left(t_{n}\right)-T_{\text {top }}\left(t_{n+1}\right)\right] \exp \left(-\frac{(\Delta t)_{n}}{R_{C} A \rho_{t} c_{p, t} L_{t}}\right)+q_{0} R_{C} A\left[1-\exp \left(-\frac{(\Delta t)_{n}}{R_{C} A \rho_{t} c_{p, t} L_{t}}\right)\right]$

Equation (20) provides the average temperature of the transducer film at any time step starting from the previous time step provided the substrate top surface temperature, $T_{\text {top }}$, is known. It may be now used in an iterative manner to obtain the temperature at the top surface of the substrate following the procedure described below:

Step 1: At any instant of time, $t_{n+1}$, the temperature at substrate top surface temperature, $T_{\text {top }}$, is guessed. A reasonable guess may be the temperature at the previous time-step.

Step 2: The BTE is next solved by assuming that the temperature of the top surface of the substrate is known and has a value equal to the value guessed in Step 1. Furthermore, this surface is assumed to be thermalizing. Once the BTE has been solved, the outgoing heat transfer rate $\dot{Q}_{\text {out }}$ can be determined using Eq. (4).

Step 3: Equation (20) is applied to obtain the temperature of the transducer film at that same instance of time, namely $T\left(t_{n+1}\right)$.

Step 4: The equation $\dot{Q}_{\text {out }}=\left(T-T_{\text {top }}\right) / R_{C}$ is next used to compute an updated value of $T_{\text {top }}$, where $\dot{Q}_{\text {out }}$ was determined in Step 2 .

Step 5. The energy equation is next solved and the temperatures of all cells in the computational domain are updated, as shown in Fig. 4.

Step 6: Steps 2-5 are repeated, as also shown in Fig. 4.

As is evident from the algorithm just described, the iterative determination of the temperature of the top surface of the substrate does not require additional iterations and is 
part and parcel of the overall iteration process (outer iteration in Fig. 4) required to couple the BTE with the energy equation.

One critical unknown that appears in the interface model is the contact resistance $R_{C}$. It depends on a number of factors including surface finish (asperity density and height distribution), pressure applied, how the transducer is incorporated on the substrate, whether any thermal glue is used or not, and also temperature. As such, it is difficult to describe using a fundamental model, and any empirical model would be strongly case dependent. Previous research has provided estimates for this contact resistance, and due to lack of better knowledge, a contact conductance value of $\left(R_{C} A\right)^{-1}=3 \times 10^{8} \mathrm{Wm}^{-2} \mathrm{~K}^{-1}$, as suggested by Ding and Minnich [38], is used.

\section{RESULTS AND DISCUSSION}

In this study, TDTR simulations were conducted in a 2D planar geometry discretized using a non-uniform mesh comprised of 6000 quadrilateral elements. The computational domain and mesh, along with the boundary conditions employed for the simulations are depicted in Fig. 5. The mesh was refined close to the laser spot and gradually coarsened outward. The aluminum transducer film was assumed to be $100 \mathrm{~nm}$ in thickness $[29,30,32]$. A constant density $\left(\rho_{t}\right)$ and specific heat capacity $\left(c_{p, t}\right)$ of $2700 \mathrm{~kg} / \mathrm{m}^{3}$ and $910 \mathrm{~J} / \mathrm{kg} / \mathrm{K}$, respectively, were used for the transducer metal film. The interface conductance was assumed to be $3 \times 10^{8} \mathrm{~W} / \mathrm{m}^{2} / \mathrm{K}$ [38]. The pump laser was pumped at a frequency of $80 \mathrm{MHz}$ (every $12.5 \mathrm{~ns}$ ) [32]. As described earlier, a progressively increasing variable time step size was used starting from 1 picosecond, enabling resolution of the entire $12.5 \mathrm{~ns}$ cycle with 25 time steps. From a numerical standpoint, the 
use of large time steps was made possible due to the implicit time advancement scheme used in the present calculations, which is in strong contrast with Monte Carlo schemes that generally use explicit time integration [34-37]. In order to gain a thorough understanding of the thermal transport process within the substrate, four virtual probes were placed at selected locations within the substrate, as depicted in Fig. 5. These probes are denoted by CPN (cross-plane near), CPF (cross-plane far), IPN (in-plane near) and IPF (in-plane far). The in-plane probe locations were adjusted based on the pump laser spot size, and the exact locations of the probes for all cases considered in this study are tabulated in Table 1. At the probe locations, not only is the temperature and heat flux stored (which is also done at all locations on the mesh) at all instances of time, but also the spectral distributions of the heat fluxes are recorded. The spectral distributions can provide better understanding of which phonon modes and frequencies actually contribute to thermal transport, as will be elaborated shortly. In addition, the probe laser location, i.e., the center of the laser spot (junction of symmetry line with top surface in Fig. 5) was separately monitored.

Two different parameters were considered for the current study. The first of these is the pump laser spot size (radius). Four different sizes were considered: $2.5 \mu \mathrm{m}, 5 \mu \mathrm{m}$, $15 \mu \mathrm{m}$ (baseline case), and $30 \mu \mathrm{m}$ [38]. Since this is a preliminary study, the laser flux was assumed to be uniform rather than Gaussian. This assumption does not alter the fundamental phonon transport process in the substrate. On the other hand, it removes secondary effects due to temperature variation within the laser spot and helps easier interpretation of the results. The second parameter considered in this study is the pump laser modulation frequency. The pump laser power was modulated sinusoidally using 
$P(t)=P_{0}\left(1+\sin \omega_{m} t\right)$, as shown schematically in Fig. 1 . Here $P_{0}$ is the time-averaged (baseline) power of the laser, and the modulation frequency is denoted by $\omega_{m}$. Two different modulation frequencies were considered: $13.33 \mathrm{MHz}$ (baseline case) and 6.67 MHz. As discussed by Regner et al. [48], the modulation frequency is directly related to the penetration depth of the heat wave into the substrate. The laser heat flux, $q_{L}(t)$, which appears in Eq. (15), is the power, $P(t)$, divided by the pump laser spot area. The baseline laser power was adjusted so that the transducer temperature increased by $5-10 \mathrm{~K}$ during a 1 picosecond pulse $[29,30,32]$.

The time-scales for Normal and Umklapp scattering in silicon used in this study were obtained from the work of Ward and Broido [20], and are given by:

$$
\begin{aligned}
& \tau_{N, p}^{-1}=A_{p}^{N} \omega^{2} T\left[1-\exp \left(-3 T / \theta_{D}\right)\right] \\
& \tau_{U, p}^{-1}=A_{p}^{U} \omega^{4} T\left[1-\exp \left(-3 T / \theta_{D}\right)\right]
\end{aligned}
$$

where the subscripts $N$ and $U$ stand for Normal and Umklapp processes, respectively, and the subscript $p$ stands for the polarization of the phonon, i.e., either longitudinal acoustic (LA) or transverse acoustic (TA). The constants in Eq. (21) are as follows: $A_{L A}^{N}=7.10 \times 10^{-20} \mathrm{~s} \cdot \mathrm{rad}^{-2} \cdot \mathrm{K}^{-1}, \quad A_{T A}^{N}=10.9 \times 10^{-20} \mathrm{~s} \cdot \mathrm{rad}^{-2} \cdot \mathrm{K}^{-1}, \quad A_{L A}^{U}=9.51 \times 10^{-47} \mathrm{~s}^{3} \cdot \mathrm{rad}^{-4} \cdot \mathrm{K}^{-1}$, $A_{T A}^{U}=37.8 \times 10^{-47} \mathrm{~s}^{3} \cdot \mathrm{rad}^{-4} \cdot \mathrm{K}^{-1}$. The Mathiessen rule [1] was then used to compute the overall relaxation time-scale. Optical phonons were not considered in this study because their contributions to thermal transport in silicon have been shown to be marginal [36] except at high temperature. Dispersion relationships for silicon were used from Brockhouse and Dolling [60], which were then used to compute the phonon group velocities for all frequencies and polarizations. 
The frequency space was discretized using 40 spectral intervals (or bands). 20 equally spaced bands were used between 0 and $\omega_{\text {max,TA }}$, and 20 additional equally spaced bands were used between $\omega_{\max , \mathrm{TA}}$ and $\omega_{\max , \mathrm{LA}}$. The number of bands were selected based on the findings of previous studies $[45,46]$. The angular space was discretized using 20 azimuthal angle divisions and 1 polar angle division. The fact that only 1 polar angle division was used was made possible by the fact that the geometry in question is $2 \mathrm{D}$ planar. In contrast, a 2D axisymmetric simulation would require a bare minimum of 4 polar angle divisions, resulting in at least four times the required computational time for the same mesh. Since band-wise parallelization is used in this study, 40 AMD Opteron processors (equal to the number of bands) were employed for parallel computations. The computation of two modulation cycles required close to 96 hours of wall clock time under these conditions. Due to the extreme computational requirements, this preliminary study was confined to $2 \mathrm{D}$ planar simulations rather than 2D axisymmetric simulations. While a 2D planar simulation does not exactly replicate a TDTR setup, it can still reveal all of the fundamental processes associated with phonon transport.

\subsection{Temperature and Heat Flux Response}

In order to investigate the detailed thermal response of the system, a baseline spot size (radius) of $15 \mu \mathrm{m}$ and pump laser modulation frequency of $13.33 \mathrm{MHz}$ was considered. The initial temperature of the system was set to 300K. Figure 6 shows the temperature response of the transducer film, the top surface of the substrate, and the center of the cell (control volume) adjacent to the top surface. In this particular case, the cell center is at a distance of $99.5 \mathrm{~nm}$ from the top surface of the substrate. All three temperature responses 
shown in Fig. 6(a) are along the center of the laser spot (i.e., along the symmetry line shown in Fig. 5). Also shown in Fig. 6(a) is the profile applied to modulate the laser power. The hollow circles indicate time instances when the pump laser is pumped along with the relative magnitude of the power used. Figure 6(a) shows that the transducer film attains peak temperature almost instantly. This is because it is thin and has a small thermal mass. On the other hand, a slight delay is observed before the top surface of the substrate attains peak temperature, and a further delay is observed in heating up the cell below the top surface. This is depicted clearly in Fig. 6(b), which shows an amplified view of the second last pulse starting at $175 \mathrm{~ns}$. At any instant of time, the difference in temperature between the transducer and the top surface of the substrate is due to the contact resistance, $R_{C}$, while the difference in temperature between the top surface of the substrate and the adjacent cell is due to the resistance posed by the substrate. As is evident from Fig. 6, the system temperature has not reached quasi steady state after two modulation cycles. However, as discussed earlier, due to the extreme computational requirements, the present calculations had to be terminated after two modulation cycles. The same simulation was also conducted using the Fourier law rather than the phonon BTE, and a comparison of the temperature response between the BTE and the Fourier law is shown in Fig. 7. In the Fourier Law calculations, an effective bulk thermal conductivity value of $366 \mathrm{~W} / \mathrm{m} / \mathrm{K}$, as computed using the time-scale expressions given by Eq. (21) in conjunction with the Debye theory (no dispersion), was used. Figure 7 shows that the temperature responses obtained using the BTE and the Fourier law is significantly different. With time, the responses deviate significantly from each other. At the very first pulse, the difference between the temperature decay rates is indicative of the 
difference between equilibrium and non-equilibrium phonon transport. As seen in Figure 7, the temperature decays more rapidly in the Fourier model than the BTE model. This is due to the so-called ballistic resistance [29] manifested under non-equilibrium transport conditions.

The detailed temperature responses shown in Figs. 6 and 7, especially within the substrate, cannot be measured by the probe laser. Rather, the probe laser measures the temperature response of the transducer film at pre-defined instances of time. Therefore, it is useful to also record the computed temporal temperature response at the same instances of time for quantitative comparison with experimental data. Figure 7(a) also shows a comparison of the computed temperature response of the transducer at the same instances of time as would be recorded by a probe laser. In this case, probing was done $4.49 \mathrm{~ns}$ after every power pulse. In addition to the fact that the actual temperatures computed by the two models at the probed location are different, a slight phase shift is also observed when the two probed responses are compared.

The differences in temperature predicted by the BTE and the Fourier law are best understood by comparing time snapshots of the spatial temperature distributions at various instances of time. Such a comparison is shown in Figure 8. The thermal wave appears to penetrate further into the material in the Fourier law solutions compared to the BTE solutions. This is a telltale sign of quasi-ballistic transport. The large mean free path phonons in the BTE solution rarely scatter and hence, do not contribute to the temperature gradient. In contrast, the Fourier law embodies a completely diffusive picture of phonon transport in which all phonons undergo infinite many scattering events, and energy is transferred effectively from high energy phonons to low energy phonons. 
Hence, heat is dissipated more effectively in the case of the Fourier law, resulting in a lower temperature at the top of the substrate.

Previous studies have insinuated that the manner in which the thermal conductivity is altered in the cross (axial) and in (radial) plane directions due to length scale effects is quite different $[32,38]$. In order to understand the multi-dimensional nature of phonon transport, the heat fluxes in the in-plane and cross-plane directions were processed at the four probe locations shown in Fig. 5. Figure 9 shows the temporal response of the normal heat flux for the baseline case of $13.33 \mathrm{MHz}$ modulation frequency and $15 \mu \mathrm{m}$ spot size (radius). At CPN and CPF, the fluxes shown are components in the cross-plane direction, while at IPN and IPF, the fluxes shown are components in the in-plane direction. The far probes, CPF and IPF, witness small fluxes, as expected, within the $200 \mathrm{~ns}$ time period. Furthermore, the effect of neither the pulse nor the modulation is felt at these far locations. When the two near probes are examined, it is seen that the near cross-plane probe (CPN), which is only $1.05 \mu \mathrm{m}$ below the laser spot, exhibits a strong effect of the pulsing of the pump laser. In addition, it also modulates with the modulation frequency. On the other hand, the near in-plane probe (IPN), which is at a distance of $0.95 \mu \mathrm{m}$ from the edge of the spot, exhibits only modulation, and no pulsing effect whatsoever. These results appear to indicate that the overall heat wave spreading is controlled by the modulation of the laser rather than the pulsing.

The modulation frequency of the laser is believed to affect the penetration depth of the laser energy. Regner et al. [48] have shown through analysis of the 1D BTE that in the case of frequency domain thermo-reflectance experiments, the penetration depth is 
expected to vary inversely with the modulation frequency, and that the relationship is nonlinear. When the frequency of modulation of the laser power is high, the hot phonons travel only a short distance before the laser power is reversed and the hot phonons scatter with the cold phonons that immediately follow. This results in relatively small penetration depth. Scattering slows down the thermal wave. Figure 10 depicts the heat flux at both the cross-plane and in-plane probe locations situated far away from the spot. These results are for the baseline spot size of $15 \mu \mathrm{m}$. As expected, initially, the heat flux is larger for the low frequency case at a given location. However, with time, as accumulation effects manifest themselves, the heat fluxes for both frequencies exhibit similar values. Also worth noting is the fact that the cross-plane heat fluxes are always larger than the in-plane fluxes for both frequencies, indicative of the different rates of heat (phonon) transfer in the two different directions.

\subsection{Thermal Conductivity}

As mentioned earlier, the probe laser in a TDTR experiment measures the transducer temperature only at certain instances of time. It does not measure the complete temperature decay of a single laser pulse. Therefore, any approach that attempts to extract the thermal conductivity by fitting Fourier law solutions to a temperature decay curve is misleading. Instead, the fit must be done to the temperature measured by the probe laser. Typically, the probed instances lag the pump laser by a few nanoseconds. A literature survey revealed that the time delay can range between $1-10$ ns $[30,32,61]$. In this study, two different time delays were considered to investigate if this delay has any impact on the extracted (fitted) thermal conductivity: 1.14 ns and 4.49 ns. For each case, several 
Fourier law simulations of the exact TDTR setup were conducted, in which the thermal conductivity used in the Fourier law simulations was changed in steps of $1 \mathrm{~W} / \mathrm{m} / \mathrm{K}$. The temperature signals were obtained from all these Fourier law simulations at the same instances of time as a probe laser would measure, i.e., either after $1.14 \mathrm{~ns}$ after the pulse or $4.49 \mathrm{~ns}$ after the pulse. These signals were then compared with the same signal obtained using the BTE simulation for the same case. The best fit was identified by computing the least square error between the two signals. Figure 11 shows the fits obtained with two different time delays for the baseline case of $13.33 \mathrm{MHz}$ modulation frequency and spot size (radius) of $15 \mu \mathrm{m}$. It is seen that when the temperature signal is probed has a significant impact on the fitted (extracted) thermal conductivity. With a 1.14 ns time delay, the fitted thermal conductivity was found to be $241 \mathrm{~W} / \mathrm{m} / \mathrm{K}$, while with a time delay of $4.49 \mathrm{~ns}$, the fitted value was found to be $321 \mathrm{~W} / \mathrm{m} / \mathrm{K}$. The computed theoretical bulk value of the thermal conductivity using the Ward and Broido [20] timescales [Eq. (21)] at $300 \mathrm{~K}$ is $366 \mathrm{~W} / \mathrm{m} / \mathrm{K}$. Hence, on the positive front, both fits indicate a suppression of the thermal conductivity due to size effects. However, depending on the probed instance used, the degree of suppression is found to be quite different. This discrepancy could be a result of the fact that the fit was conducted on a signal that has not reached quasi steady state yet, and therefore, the quality of the fit is poor. Nonetheless, this is an issue that is noteworthy for future researchers.

The fitted (extracted) values of the thermal conductivity for both time delays and for all parametric variations are shown in Table 2. As discussed earlier, the chosen time delay affects the fit and the value of the extracted thermal conductivity. However, in both cases, two important trends are observed. First, the thermal conductivity does not change 
significantly with a change in the modulation frequency. These predictions agree with past experimental works, which reported negligible frequency dependence of the thermal conductivity at room temperature [30] as well as at low temperature [29]. The second point to note from the data shown in Table 2 is that the thermal conductivity is relatively unchanged for large spot sizes, and eventually decreases as the spot size is decreased significantly. This behavior of the thermal conductivity with spot size has also been observed experimentally by Minnich et al. [29]. The fact that the modulation frequency does not have significant impact on the thermal conductivity may be inferred from the het flux plots shown in Fig. 10. As explained earlier, although the initial heat flux evolution is different for the two frequencies, after the first modulation cycle, the two heat flux signals, although out of phase, go almost hand in hand magnitude wise. In the case of the spot size variation, decrease in the spot size changes the transport from almost onedimensional transport to two-dimensional transport. As a result, the resistance posed to the transport of phonons is slightly larger, resulting in a lower thermal conductivity.

One of the critical features of non-equilibrium transport in a real material is that carriers have a wide range of mean free paths. As a result, while some phonons may be in the diffusive regime of transport, others are in the ballistic regime. Consequently, they contribute to the thermal transport in different ways. It is now widely believed that the experimentally observed behavior of thermal conductivity suppression at the nanonscale is due to the fact that phonons of certain frequency (or mean free path) do not contribute to the thermal conductivity. In order to assess this claim, the cumulative thermal conductivity distribution function was constructed. In normalized form, this is written as 
$\kappa_{a c c}\left(\Lambda^{*}\right)=\frac{\kappa\left(\Lambda^{*}\right)}{\kappa_{b u l k}}=\frac{\frac{1}{3} \int_{\Lambda_{\min }}^{\Lambda^{*}} c_{\Lambda}\left|\mathbf{v}_{\Lambda}\right| \Lambda d \Lambda}{\frac{1}{3} \int_{\Lambda_{\min }}^{\Lambda_{\max }} c_{\Lambda}\left|\mathbf{v}_{\Lambda}\right| \Lambda d \Lambda}$

where $c_{\Lambda}$ is the specific heat capacity per unit volume per unit length, and $\boldsymbol{v}_{\Lambda}$ is the group velocity of the phonons as a function of the mean free path, $\Lambda$. Figure 12 shows the normalized cumulative thermal conductivity as a function of the phonon mean free path, as computed using Eq. (22). According to this plot, the bulk thermal conductivity is manifested when phonons of all mean free path are considered. In other words, all phonons contribute to the thermal conductivity. Figure 12 also shows the thermal conductivity extracted by fitting the BTE results with the Fourier solutions using two different time delays for a spot size (radius) of $15 \mu \mathrm{m}$ and modulation frequency of 13.33 MHz. This value of thermal conductivity is manifested at mean free path values that are considerably smaller than the maximum possible mean free path. This implies that the largest mean free paths (to the right of the vertical lines) do not contribute to the thermal conductivity - the so-called size effect. The experimental data reported by Minnich et al. [29] demonstrate suppression of the thermal conductivity $\left(\kappa / \kappa_{\text {bulk }}\right)$ in the range 0.6 to 0.8, which is in agreement with the computed results shown in Fig. 12 and Table 2.

\section{SUMMARY AND CONCLUSIONS}

The thermal conductivity of most semiconductors is size dependent at the sub-micron scale. The size dependence of the thermal conductivity can be utilized to tailor its value provided the mean free path spectrum of the dominant energy carrier, namely phonons, is known. While several theoretical techniques have been used to compute the mean free 
path, the computed mean free paths have never been validated directly against measured data. In the past decade, so-called pump probe experimental techniques have made considerable progress. One of these techniques is the so-called Time Domain ThermoReflectance (TDTR) technique. The data collected by TDTR experiments can be used to validate theoretically computed spectral mean free paths. However, since the experiments do not directly measure the mean free path spectrum, but, rather, temperature (through altered transducer reflectance), an intervening model is necessary to relate the mean free path spectrum to the temperature. It is now widely accepted that the most appropriate model to this end is the BTE for phonons. The BTE for phonons, even under the single relaxation time approximation, is a seven-dimensional (six-dimensional if a spatially twodimensional computational domain is considered) partial differential equation, making it extremely challenging to solve.

In this study, BTE computations are conducted in a two-dimensional geometry to simulate the conditions of a TDTR experimental setup. Silicon is used as the candidate substrate material, while aluminum is used as the transducer material. The thin aluminum transducer film is modeled using the Fourier law under the lumped mass approximation, and coupled to the BTE calculations in the silicon substrate using a self-consistent iterative procedure. Parametric variations considered are the pump laser's modulation frequency, and the laser spot size (radius). The BTE computations are performed with a computational mesh comprised of 6000 quadrilateral control volumes. For angular discretization, 20 azimuthal angles are used, while for discretization of the frequency space, 40 spectral intervals (or bands) are used. For time advancement, the backward Euler procedure with unequal time step size is used, with the smallest time step size being 
1 ps. Each simulation is carried out until $200 \mathrm{~ns}$ of total time, and requires approximately 96 hours of wall clock time on a parallel system with 40 processors. The same simulations are repeated using the Fourier law of heat conduction with different thermal conductivity values, and the least-square error between the BTE results and the Fourier results, as measured by the probe laser, is minimized to extract effective thermal conductivities. It is demonstrated that the instance of probing (time delay after the power pulse) plays a significant role in determining the fitted (extracted) value of the thermal conductivity. Size effects are clearly manifested by the BTE computations. It is found that the thermal conductivity value is suppressed by size effects, with the actual thermal conductivity varying between $0.6-0.8$ times the bulk value. The modulation frequency did not have a significant impact on the thermal conductivity, while the thermal conductivity was found to decrease with decrease in spot size after an initial plateau at large spot sizes. Both of these trends agree with previously reported experimental observations, and the physical reasons for these trends have been delineated. The computations reported in this study constitute the first critical step toward validating theoretically computed mean free path spectrum directly against experimental measurements, or conversely, to extract it from experimental measurements.

\section{ACKNOWLEDGMENTS}

Financial support for this work was provided, in part, by the National Science Foundation's Computation and Data-Enabled Science and Engineering (CDESE) Program through Award Number 1250215. The Ohio Supercomputer Center is 
acknowledged for providing computational resources through Grant Number PAS0588-2.

ESI Group is acknowledged for providing free licenses to CFD-ACE $+{ }^{\mathrm{TM}}$. 


\section{REFERENCES}

[1] Tien, C.L., Majumdar, A., and Gerner, F.M., eds., 1998, Microscale Energy Transport, Taylor and Francis.

[2] Zhang, Z., (2007), Nano/Microscale Heat Transfer, McGraw Hill, New York.

[3] Ju, Y.S., and , Goodson, K. E., (1999), "Phonon Scattering in Silicon Films with Thickness of Order 100 nm," Appl. Phys. Lett., 74, No. 20, pp. 3005-3007, DOI: 10.1063/1.123994

[4] Cahill, D. G., Ford, W. K., Goodson, K. E., Mahan, G. D., Majumdar, A.,Maris, H. J., Merlin, R., and Phillpot, S. R., (2003), "Nanoscale Thermal Transport," Journal of Applied Physics, Vol. 93(2), pp. 793-818. DOI: 10.1063/1.1524305

[5] Regner, K.T., Freedman, J.P., and Malen, J.A., (2015), "Advances in Studying Phonon Mean Free Path Dependent Contributions to Thermal Conductivity," Nanoscale and Microscale Thermophysical Engineering, Vol. 19, pp. 183-205.

[6] Callaway, J., (1959), "Model for lattice thermal conductivity at low temperatures," Physical Review, Vol. 113(4), pp. 1046-1051.

[7] Holland, M.G., (1963), "Analysis of lattice thermal conductivity," Physical Review, Vol. 132(6), pp. 2461-2471.

[8] Holland, M.G., (1964), "Phonon scattering in semiconductors from thermal conductivity studies," Physical Review, Vol. 134(2A), pp. A471-A480.

[9] Klemens, P.G., (1969), "Theory of thermal conductivity of solids," In Thermal Conductivity, Vol. 1, Academic Press, London, pp.1-68

[10] Lukes, J. R., Li, D. Y., Liang, X.-G., and Tien, C.-L., (2000), "Molecular dynamics study of solid thin-film thermal conductivity," Journal of Heat Transfer, Vol. 122(3), pp. 536-543.

[11] Volz, S., Saulnier, J.B, Chen, G., and Beauchamp, P., (2000), "Computation of thermal conductivity of si/ge superlattices by molecular dynamics techniques," Micro-electronics Journal, Vol. 31(9-10), pp. 815-819.

[12] Heino, P., (2004), "Nanoscale thermal conductivity: Size dependence by molecular dynamics," Physica Scripta, Vol. 2004(T114), pp. 171.

[13] McGaughey, A.J.H., and Kaviany, M., (2006), "Phonon transport in molecular dynamics simulations: Formulation and thermal conductivity prediction," Chapter 2 in. Advances in Heat Transfer, Vol. 39, pp. 169-225.

[14] Henry, A.S., and Chen, G., (2008), "Spectral phonon transport properties of silicon based on molecular dynamics simulations and lattice dynamics," Journal of Computational and Theoretical Nanoscience, Vol. 5, pp. 141-152, February 2008.

[15] Omini, M. and Sparavigna, A., (1995), "An iterative approach to the phonon Boltzmann-equation in the theory of thermal-conductivity," Physica B, Vol. 212, pp. $101-112$

[16] Omini, M. and Sparavigna, A., (1996), "Beyond the isotropic-model approximation in the theory of thermal conductivity," Physical Review B, Vol. 53, pp. $9064-9073$

[17] Omini, M. and Sparavigna, A., (1997), "Heat transport in dielectric solids with diamond structure," Nuovo Cimento D, Vol. 19, pp. 1537-1563

[18] Mittal, A., and Mazumder, S., (2010), "Monte Carlo Study of Phonon Heat Conduction in Silicon Thin Films Including Contributions of Optical Phonons," Journal of Heat Transfer, Vol. 132, Article number 052402. 
[19] Broido, D.A., Malorny, M., Birner, G., Mingo, N., and Stewart, D.A., (2007), "Intrinsic lattice thermal conductivity of semiconductors from first principles," Applied Physics Letters, Vol. 91(23), pp. 231922.

[20] Ward, A., and Broido, D.A., (2010), "Intrinsic phonon relaxation times from first principles studies of the thermal conductivities of Si and Ge," Physical Review B, Vol. 81(8), pp. 085205.

[21] Deinzer, G., Birner, G. and Strauch, D., (2003), "Ab initio calculation of the linewidth of various phonon modes in germanium and silicon," Physical Review B, Vol. 67, Art. No. 144304.

[22] Giannozzi, P., Degironcoli, S., Pavone, P. and Baroni, S., (1991), "Ab initio calculation of phonon dispersions in semiconductors," Physical Review B, Vol. 43, pp. 7231-7242

[23] Baroni, S., de Gironcoli, S., Dal Corso, A. and Giannozzi, P., (2001), "Phonons and related crystal properties from density-functional perturbation theory," Rev. Mod. Phys., Vol. 73, pp. 515-562

[24] Debernardi, A., Baroni, S. and Molinari, E., (1995), "Anharmonic phonon lifetimes in semiconductors from density-functional perturbation-theory," Physical Review Letters, Vol. 75, pp. 1819-1822

[25] Ma, J., Delaire, O., May, A.F., Carlton, C.E., McGuire, M.A., VanBebber L.H., Abernathy, D.L., Ehlers, G., Hong, T., Huq, A., Tian, W., Keppens, V.M., ShaoHorn, Y., and Sales, B.C., (2013), "Glass-like Phonon Scattering from a Spontaneous Nanostructure in AgSbTe2," Nature Nanotechnology, Vol. 8, pp. 445451.

[26] Shukla, A., Calandra, M., d'Astuto, M., Lazzeri, M., Mauri, F., Bellin, C., Krisch, M., Karpinski, J., Kazakov, S.M., Jun, J., Daghero, D., and Parlinski, K., (2003), "Phonon Dispersion and Lifetimes in MgB2," Physical Review Letters, Vol. 90, Article number 095506.

[27] Cuffe, J., Eliason, J.K., Maznev, A.A., Collins, K.C., Johnson, J.A., Shchepetov, A., Prunnila, M., Ahopelto, J., Sotomayor Torres, C.M., Chen, G., and Nelson, K.A., (2015), "Reconstructing phonon mean-free-path contributions to thermal conductivity using nanoscale membranes," Physical Review B, Vol. 91, Article number 245423.

[28] Johnson, J.A., Maznev, A.A., Cuffe, J., Eliason, J.K., Minnich, A.J., Kehoe, T., Sotomayor Torres, C.M., Chen, G., and Nelson, K.A., (2013), "Direct Measurement of Room-Temperature Nondiffusive Thermal Transport Over Micron Distances in a Silicon Membrane," Physical Review Letters, Vol. 110, Article Number 025901.

[29] Minnich, A.J., Johnson, J.A., Schmidt, A.J., Esfarjani, K., Dresselhaus, M.S., Nelson, K.A., and Chen, G., (2011), "Thermal Conductivity Spectroscopy Technique to Measure Phonon Mean Free Paths," Physical Review Letters, Vol. 107, pp. 095901.

[30] Koh, Y.K., and Cahill, D.G., (2007), "Frequency Dependence of the Thermal Conductivity of Semiconductor Alloys," Physical Review B, Vol. 76, pp. 075207.

[31] Cahill, D.G., and Watanabe, F., (2004), "Thermal Conductivity of Isotropically Pure and Ge-doped Si Epitaxial Layers from 300 to 550K," Physical Review B, Vol. 70, Article number 235322. 
[32] Schmidt, A.J., Chen, X., and Chen, G., (2008), "Pulse accumulation, radial heat conduction, and anisotropic thermal conductivity in pump-probe transient thermoreflectance," Review of Scientific Instruments, Vol. 79, Article number 114902.

[33] Norris, P.M., Caffrey, A.P., Stevens, R.J., Klopf, J.M., McLeskey Jr., J.T., and Smith, A.N., (2003), "Femtosecond pump-probe nondestructive examination of materials," Review of Scientific Instruments, Vol. 74, pp. 400.

[34] Mazumder, S., and Majumdar, A., (2001), "Monte Carlo Study of Phonon Transport in Solid Thin Films Including Dispersion and Polarization," Journal of Heat Transfer, Vol. 123, pp. 749-759, DOI: 10.1115/1.1377018

[35] Lacroix, D., Joulain, K., and Lemonnier, D. (2005), "Monte Carlo transient phonon transport in Silicon and Germanium at nanoscale," Phys. Rev. B, Vol. 72, 064305(111), DOI: 10.1103/PhysRevB.72.064305

[36] Mittal, A., and Mazumder, S., (2010), "Monte Carlo Study of Phonon Heat Conduction in Silicon Thin Films Including Contributions of Optical Phonons," Journal of Heat Transfer, Vol. 132, Article number 052402.

DOI: $10.1115 / 1.4000447$

[37] Peraud, J.-P.M., and Hadjiconstantinou, N.G., (2011) "Efficient simulation of multidimensional phonon transport using energy-based variance-reduced Monte Carlo formulations," Physical Review B, Vol. 84, Art. No. 205331

[38] Ding, D., Chen, X., Minnich, A.J., (2014), "Radial Quasiballistic Transport in Time-Domain Thermo-Reflectance Studied Using Monte Carlo Simulations," Applied Physics Letters, Vol. 104, p. 143104.

[39] Murthy, J.Y., and Mathur, S.R., (2002), "Computation of Sub-Micron Thermal Transport Using an Unstructured Finite-Volume Method," Journal of Heat Transfer, Vol. 124, pp. 1176-1181

[40] Narumanchi, S.V.J., (2003), "Simulation of heat transport in sub-micron conduction", PhD. Dissertation, Department of Mechanical Engineering, Carnegie Mellon University.

[41] Narumanchi, S.V.J., Murthy, J.Y., Amon, C.H., (2004), "Sub-micron heat transport model in silicon accounting for phonon dispersion and polarization," Journal of Heat Transfer, Vol. 126, pp. 946-955

[42] Murthy, J.Y., Narumanchi, S.V.J., Pascual-Gutierrez, J.A., Wang, T., Ni, C. and Mathur, S.R., (2005) "Review of Multi-Scale Simulation in Sub-Micron Heat Transfer", International Journal for Multiscale Computational Engineering, Vol. 3, pp. 5-32

[43] Ni, C., and Murthy, J.Y., (2009), "Parallel Computation of the Phonon Boltzmann Transport Equation," Numerical Heat Transfer, Part B, Vol. 55, pp. 435-456.

DOI: $10.1080 / 10407780902864771$

[44] Mittal, A., and Mazumder, S., (2011), "Generalized Ballistic-Diffusive Formulation and Hybrid $\mathrm{S}_{\mathrm{N}}-\mathrm{P}_{\mathrm{N}}$ Solution of the Boltzmann Transport Equation for Phonons for Non-Equilibrium Heat Conduction," Journal of Heat Transfer, Vol. 133(9), Article No. 092402 , DOI: $10.1115 / 1.4003961$

[45] Mittal, A., and Mazumder, S., (2011), "Hybrid Discrete Ordinates-Spherical Harmonics Solution to the Boltzmann Transport Equation for Phonons for NonEquilibrium Heat Conduction," Journal of Computational Physics, Vol. 230(18), pp. 6977-7001, DOI: 10.1016/j.jcp.2011.05.024 
[46] Ali, S.A., Kollu, G., Mazumder, S., Sadayappan, P., and Mittal, A., (2014), "LargeScale Parallel Computation of the Phonon Boltzmann Transport Equation," International Journal of Thermal Sciences, Vol. 86, pp. 341-351. DOI: 10.1016/j.ijthermalsci.2014.07.019

[47] Ali, S.A., and Mazumder, S., (2015), "Phonon Heat Conduction in MultiDimensional Heterostructures: Predictions using the Boltzmann Transport Equation," Journal of Heat Transfer, Vol. 137(10), Article No. 102401.

[48] Regner, K.T., McGaughey, A.J.H., and Malen, J.A., (2014), "Analytical Interpretation of Nondiffusive Phonon Transport in Thermoreflectance Thermal Conductivity Measurements," Physical Review B., Vol. 90, p. 064302

[49] Minnich, A.J., (2012), "Determining Phonon Mean Free Paths from Observations of Quasiballistic Thermal Transport," Physical Review Letters, Vol. 109, p. 205901.

[50] Hua, C., and Minnich, A.J., (2014), "Transport Regimes in Quasiballistic Heat Conduction," Physical Review B, Vol. 89, p. 094302.

[51] Majumdar, A., (1993), "Microscale Heat Transfer in Dielectric Thin Films," Journal of Heat Transfer, Vol. 115, pp. 7-16. DOI: 10.1115/1.2910673

[52] Whitaker, S., (1983), Fundamental Principles of Heat Transfer, Krieger Publishing Company.

[53] Modest M.F., (2013), Radiative Heat Transfer, Third Edition, Academic Press.

[54] Hua, C., Chen, X., Ravichandran, N.K., and Minnich, A.J., (2015), "Fresnel Transmission Coefficients for Thermal Phonons at Solid Interfaces," arXiv:1509.07806

[55] Mazumder, S., (2016), Numerical Methods for Partial Differential Equations: Finite Difference and Finite Volume Methods, First Edition, Academic Press, New York.

[56] Raithby, G.D., and Chui, E.H., (1990), "A Finite-Volume Method for Predicting a Radiant Heat Transfer in Enclosures with Participating Media," Journal of Heat Transfer, Vol. 112(2), pp. 415-423. DOI: 10.1115/1.2910394

[57] Chai, J.C., Lee, H.S. and Patankar, S.V., (1994), "Finite-Volume Method for Radiative Heat Transfer," Journal of Thermophysics and Heat Transfer, Vol. 8, pp. 419-425, DOI: $10.2514 / 3.559$

[58] Chai, J.C., Lee, H.S., and Patankar, S.V., (1993), "Ray effect and false scattering in the discrete ordinates method," Numerical Heat Transfer, Part B, Vol. 24, pp. 373389, DOI: 10.1080/10407799308955899

[59] Saad, Y., (2003), Iterative Methods for Sparse Linear Systems, $2^{\text {nd }}$ Edition, SIAM, Philadelphia, PA.

[60] Brockhouse, B. N., (1959), "Lattice Vibrations in Silicon and Germanium", Phys. Rev. Lett., Volume 2(6), pp. 256-258. DOI: 10.1103/PhysRevLett.2.256

[61] Zeng. L, (2013), Experimental and Numerical Investigation of Phonon Mean Free Path Distribution, Ph.D. Dissertation, Massachusetts Institute Of Technology 


\section{NOMENCLATURE}

$\begin{array}{ll}c & \text { specific heat capacity per unit volume }\left[\mathrm{J} \mathrm{m}^{-3} \mathrm{~K}^{-1}\right] \\ D & \text { density of states per unit volume }\left[\mathrm{m}^{-3}\right] \\ f & \text { number density function } \\ f_{0} & \text { equilibrium number density function } \\ G_{\omega, p} & \text { spectral directionally integrated intensity }\left[\mathrm{Wm}^{-2} \mathrm{rad}^{-1} \mathrm{~s}\right] \\ \hbar & \text { Dirac (or reduced Planck) constant }=1.0546 \times 10^{-34}\left[\mathrm{~m}^{2} \mathrm{~kg} . \mathrm{s}^{-1}\right] \\ I_{\omega, p} & \text { spectral directional phonon intensity }\left[\mathrm{Wm}^{-2} \mathrm{sr}^{-1} \mathrm{rad}^{-1} \mathrm{~s}\right] \\ I_{0, \omega, p} & \text { equilibrium phonon intensity }\left[\mathrm{Wm}^{-2} \mathrm{sr}^{-1} \mathrm{rad}^{-1} \mathrm{~s}\right] \\ k_{B} & \text { Boltzmann constant }=1.381 \times 10^{-23}\left[\mathrm{~m}^{2} \mathrm{~kg}^{-2} \mathrm{~s}^{-2}\right] \\ \hat{\mathbf{n}} & \text { unit surface normal vector } \\ N_{b a n d} & \text { total number of spectral intervals }(\mathrm{or} \mathrm{bands)} \\ N_{c e l l} & \text { number of control volumes (or cells) } \\ N_{d i r} & \text { number of control angles (or directions) } \\ p & \text { phonon polarization index } \\ \mathbf{q} & \text { heat flux vector }\left[\mathrm{Wm}{ }^{-2}\right] \\ \dot{Q} & \text { heat transfer rate }[\mathrm{W}] \\ \mathbf{r} & \text { position vector }[\mathrm{m}] \\ R_{C} & \text { contact resistance }\left[\mathrm{KW}{ }^{-1}\right] \\ \hat{\mathbf{s}} & \text { unit direction vector } \\ t & \text { time [s] } \\ T & \text { absolute temperature }[\mathrm{K}] \\ u & \text { internal energy per unit volume }\left[\mathrm{J} \mathrm{m}^{-3}\right] \\ V_{k} & \text { volume of cell } k\left[\mathrm{~m}{ }^{3}\right] \\ & \end{array}$

\section{Greek}

$\begin{array}{ll}\alpha & \text { degree of specularity } \\ \theta & \text { polar angle [radians] } \\ \kappa & \text { thermal conductivity }\left[\mathrm{W} \mathrm{m}^{-1} \mathrm{~K}^{-1}\right] \\ \Lambda & \text { mean free path }[\mathrm{m}] \\ \mathbf{v}_{\omega, p} & \text { phonon group velocity vector }\left[\mathrm{m} \mathrm{s}^{-1}\right] \\ \tau_{\omega, p} & \text { spectral relaxation time scale }[\mathrm{s}] \\ \omega & \text { angular frequency }\left[\mathrm{rad} \mathrm{s}{ }^{-1}\right] \\ \Omega & \text { solid angle }(\mathrm{sr}) \\ \psi & \text { azimuthal angle }[\mathrm{rad}]\end{array}$




\section{List of Figures}

Figure 1: Schematic representation of a TDTR experimental setup showing the modulated power pulse train

Figure 2: Schematic representation of the energy flow diagram in the lumped mass model used for the transducer film

Figure 3: Unstructured stencil showing geometric connectivity and a discrete direction (line of sight) of propagation of phonons.

Figure 4: Overall solution algorithm used for TDTR simulations

Figure 5: Geometry, mesh, and boundary conditions used in TDTR simulations

Figure 6: Temperature response computed using the phonon BTE: (a) two complete modulation cycles, and (b) a single (second last) power pulse.

Figure 7: Comparison of the temperature response obtained using the phonon BTE and the Fourier law: (a) transducer film, and (b) top surface of substrate.

Figure 8: Temperature distributions in the silicon substrate predicted by the Fourier law and the BTE at various instances of time: (a) $29.5 \mathrm{~ns}$, (b) $58.9 \mathrm{~ns}$, (c) $90.7 \mathrm{~ns}$, and (d) $118.8 \mathrm{~ns}$.

Figure 9: Temporal evolution of the heat flux at various probe locations for a spot size (radius) of $15 \mu \mathrm{m}$, and modulation frequency of $13.33 \mathrm{MHz}$.

Figure 10: Temporal evolution of the effect of modulation frequency on normal heat fluxes at the four probe locations. A spot size (radius) of $15 \mu$ was used.

Figure 11: Fits obtained to BTE solutions using the Fourier law with two different probe laser time delays: (a) 1.14 ns after power pulse, (b) 4.49 ns after power pulse.

Figure 12: Thermal conductivity accumulation function along with the computed (from BTE) thermal conductivity for a spot size (radius) of $15 \mu \mathrm{m}$ and modulation frequency of $13.33 \mathrm{MHz}$ with two different time delays for probing.

\section{List of Tables}

Table 1: Location of virtual probes placed within the computational domain (see Fig. 5)

Table 2: Computed thermal conductivity using the BTE as a function of the pump laser modulation frequency and the spot size (radius). The bulk thermal conductivity is 366 $W / m / K$. 

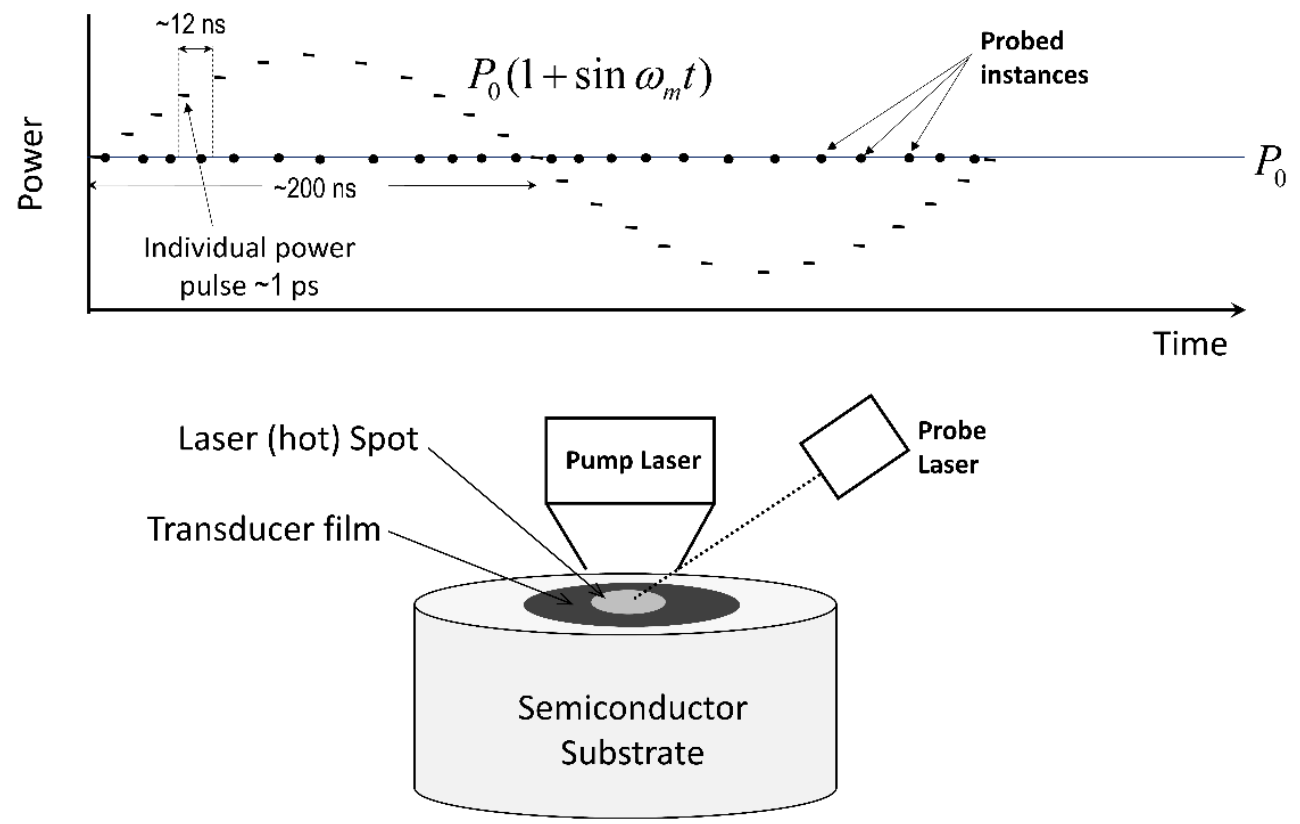

Figure 1: Schematic representation of a TDTR experimental setup showing the modulated power pulse train 


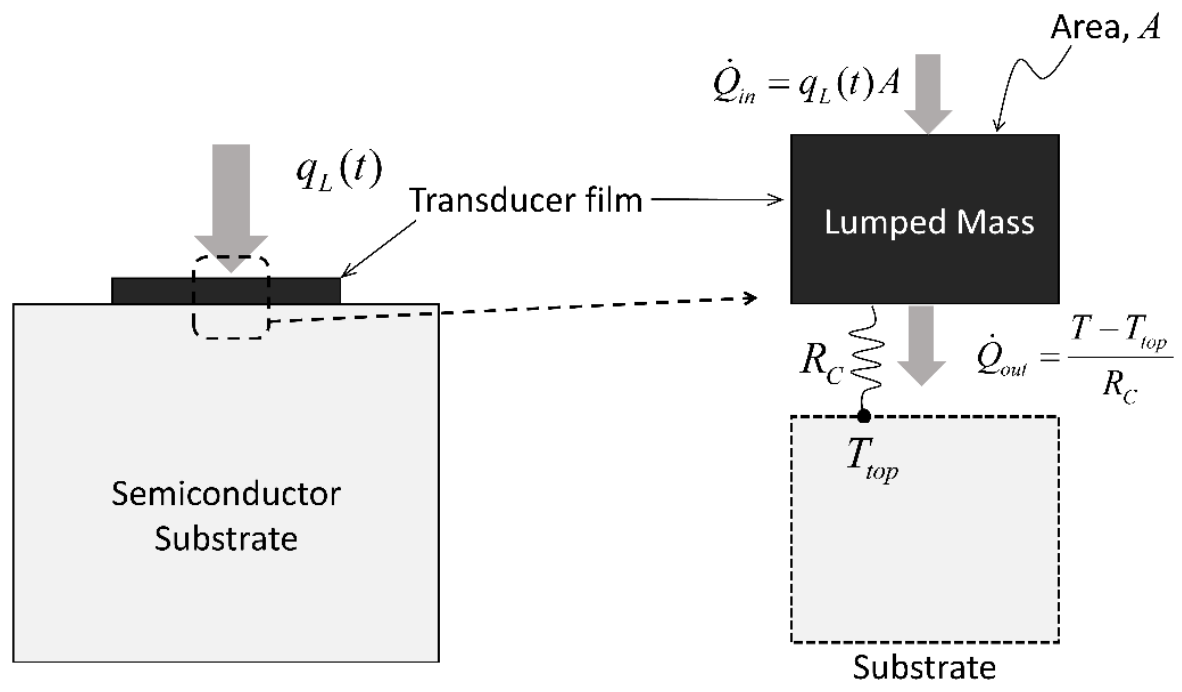

Figure 2: Schematic representation of the energy flow diagram in the lumped mass model used for the transducer film 


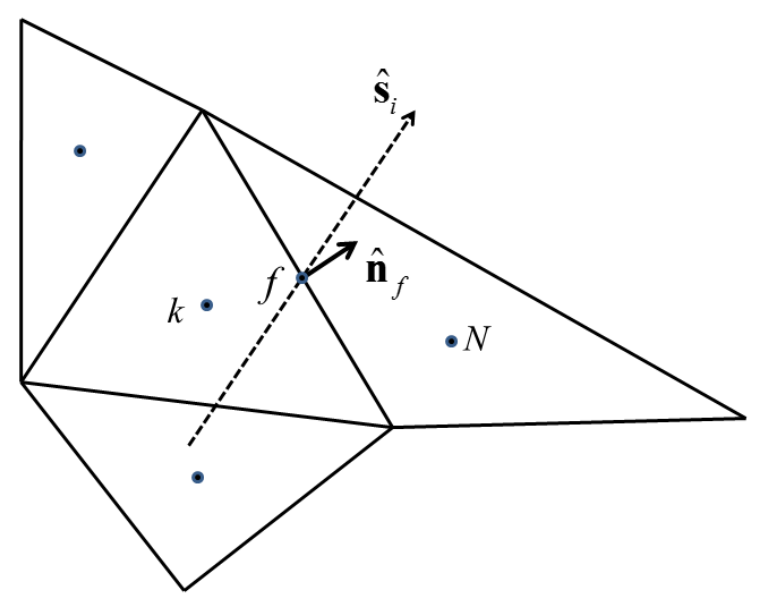

Figure 3: Unstructured stencil showing geometric connectivity and a discrete direction (line of sight) of propagation of phonons. 


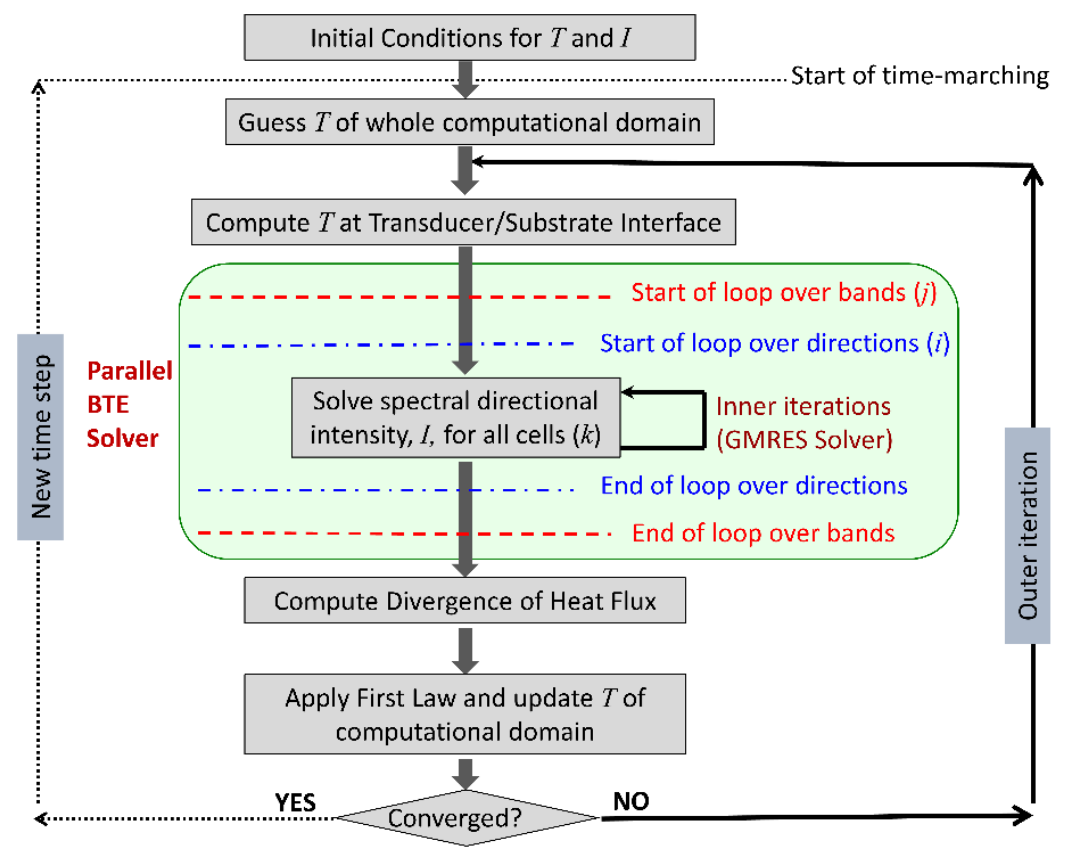

Figure 4: Overall solution algorithm used for TDTR simulations 


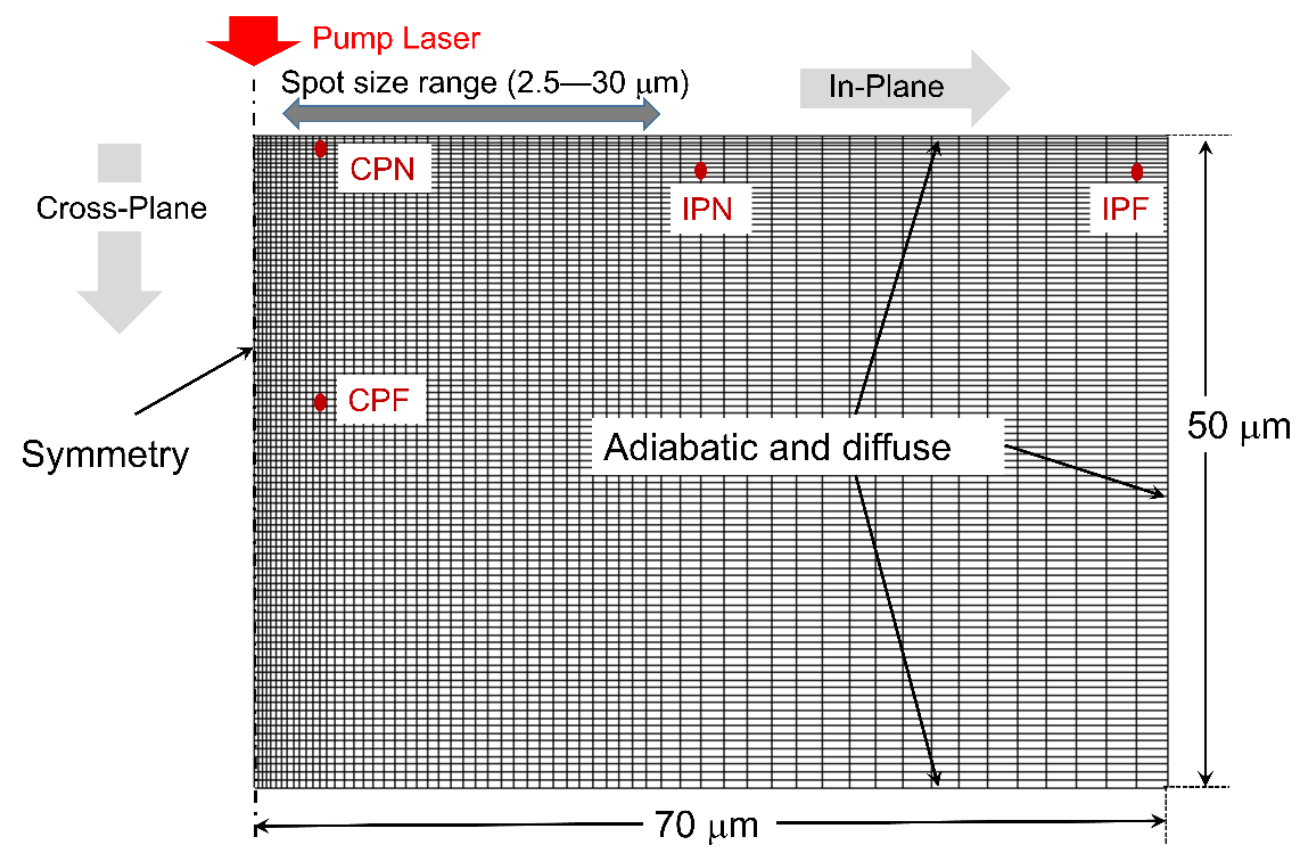

Figure 5: Geometry, mesh, and boundary conditions used in TDTR simulations 


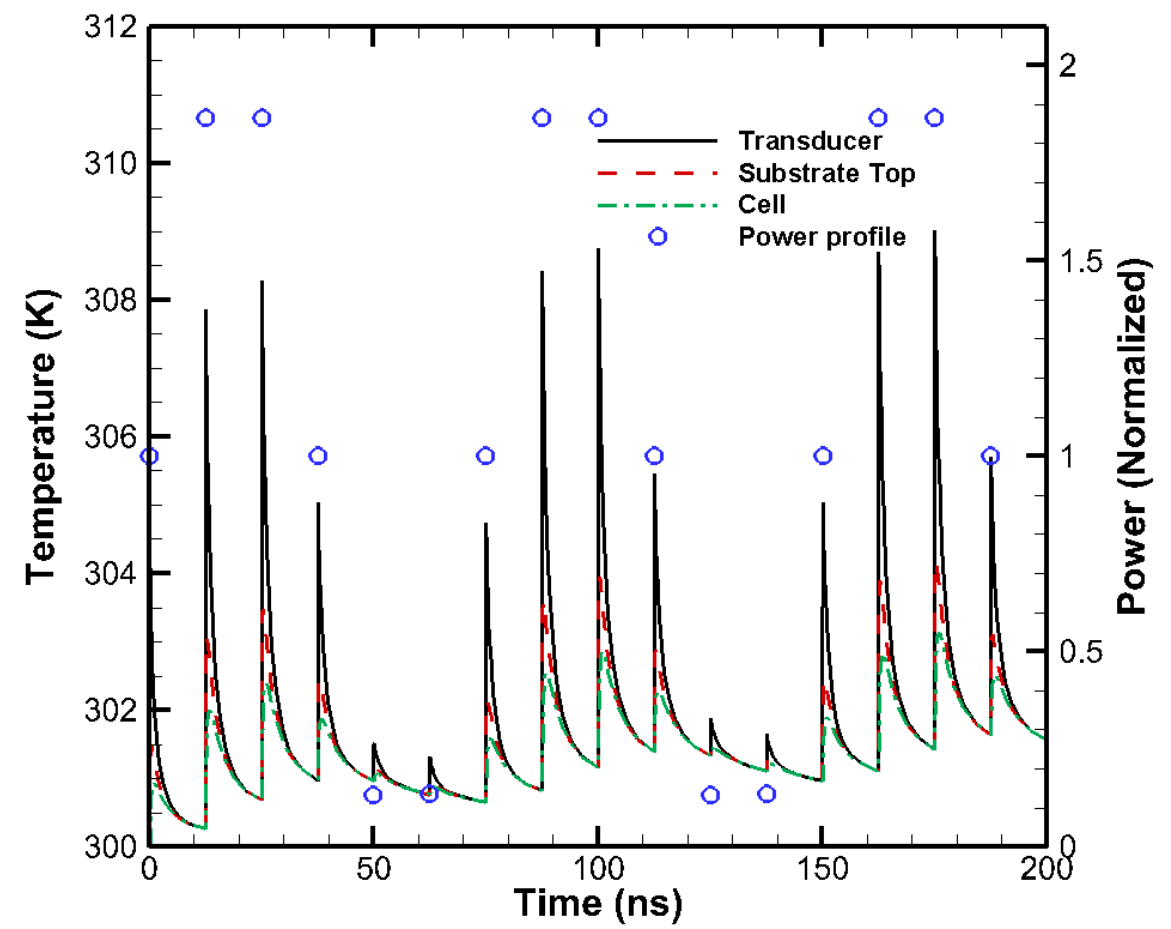

(a)

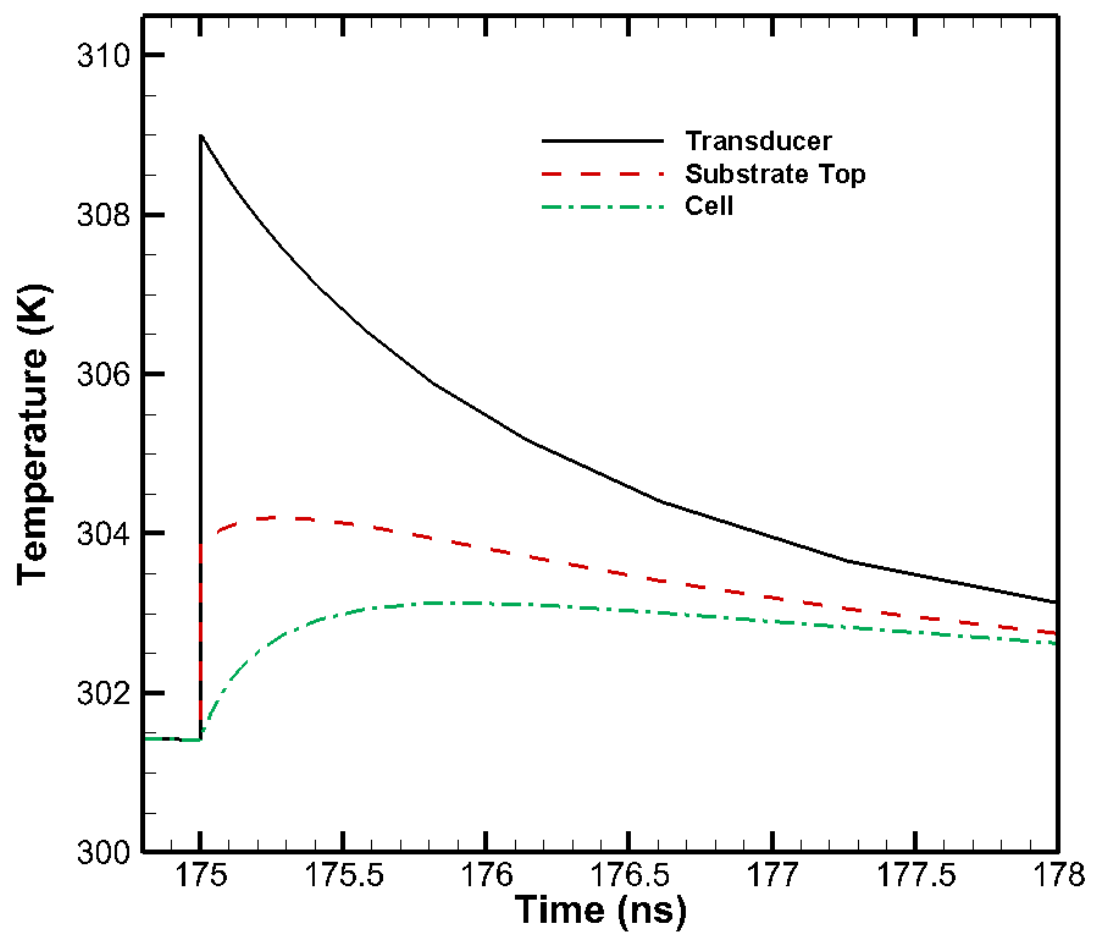

(b)

Figure 6: Temperature response computed using the phonon BTE: (a) two complete modulation cycles, and (b) a single (second last) power pulse. 


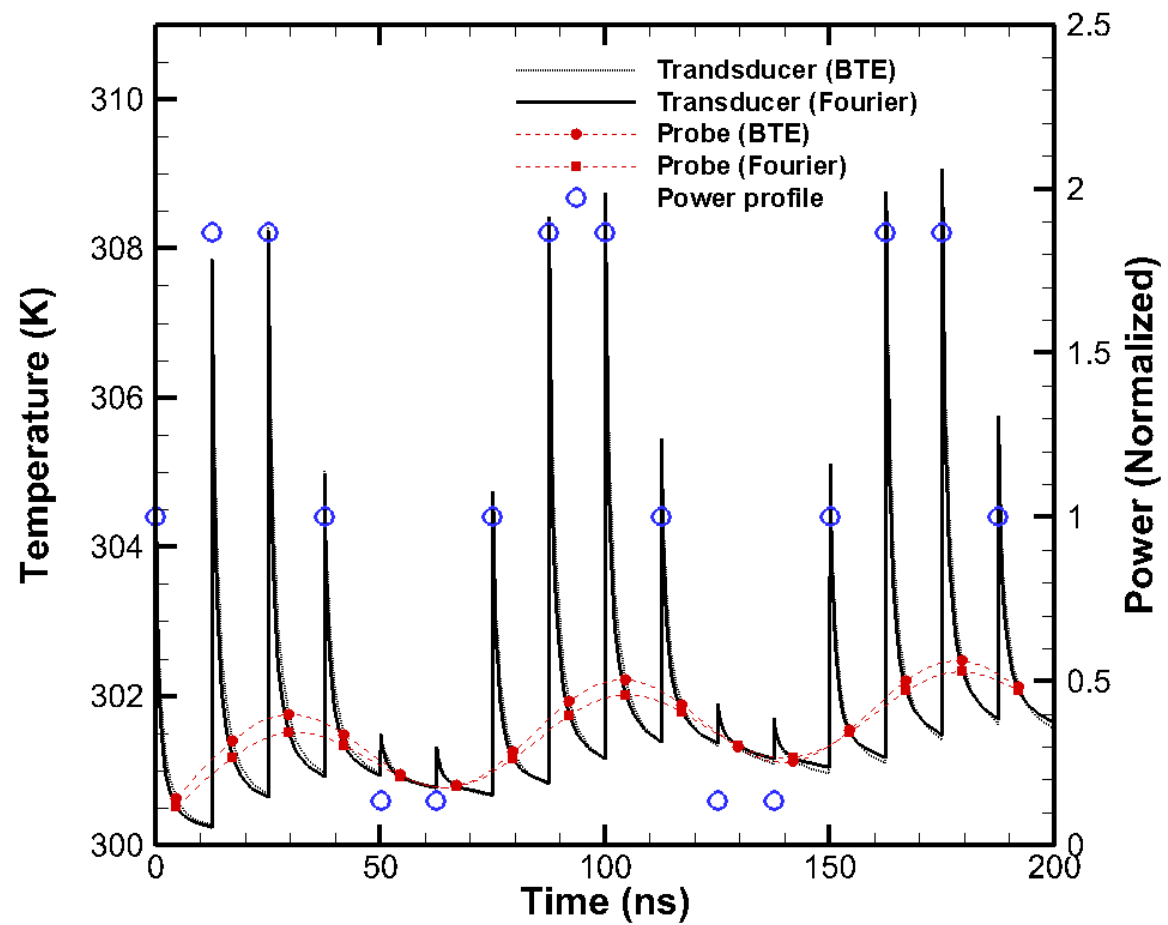

(a)

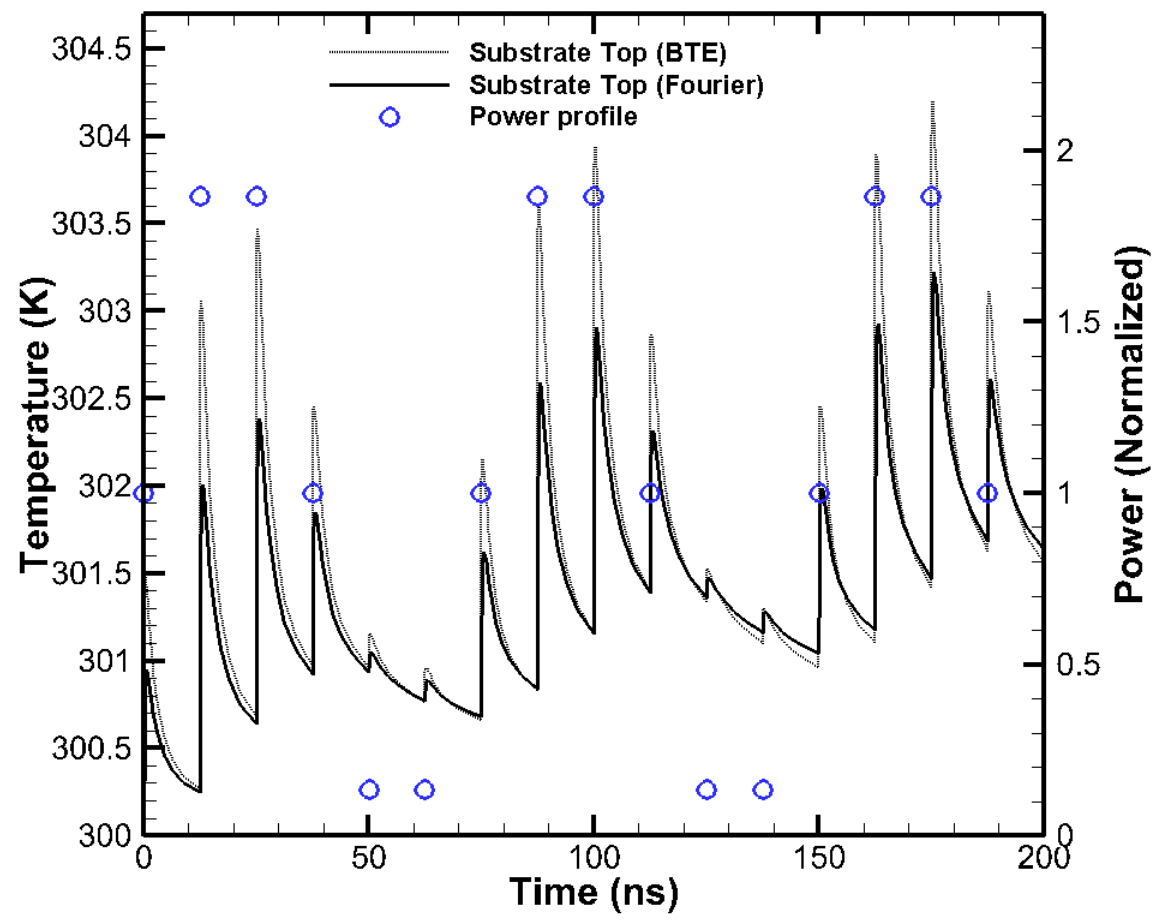

(b)

Figure 7: Comparison of the temperature response obtained using the phonon BTE and the Fourier law: (a) transducer film, and (b) top surface of substrate. 


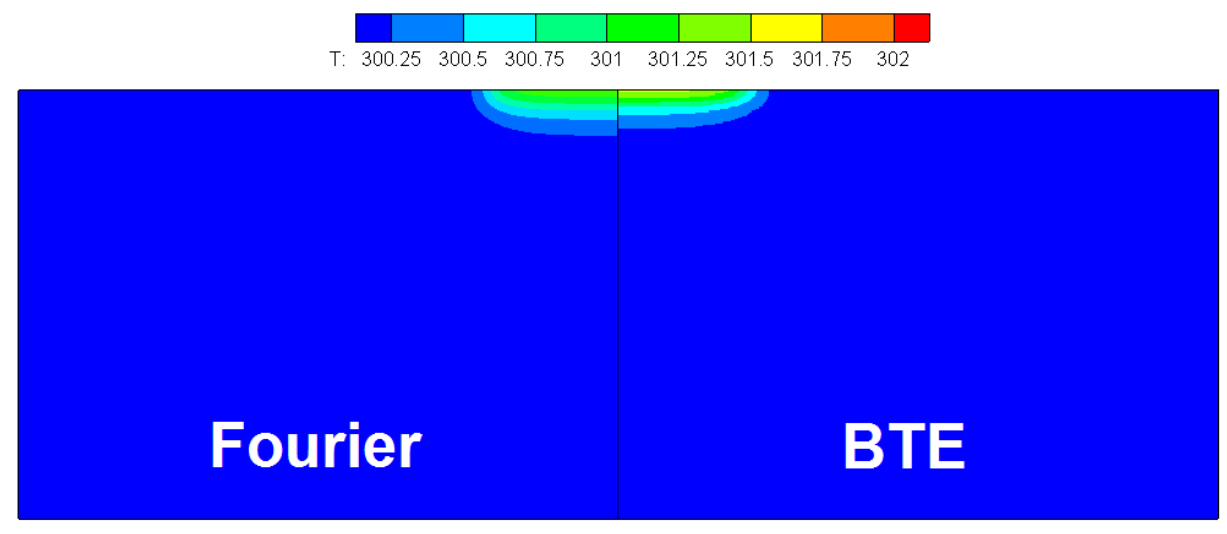

(a)

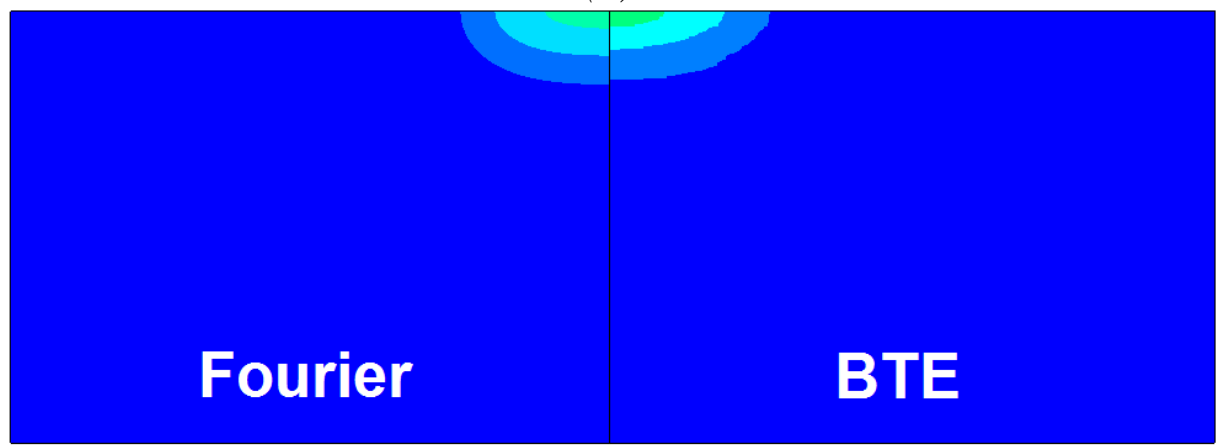

(b)

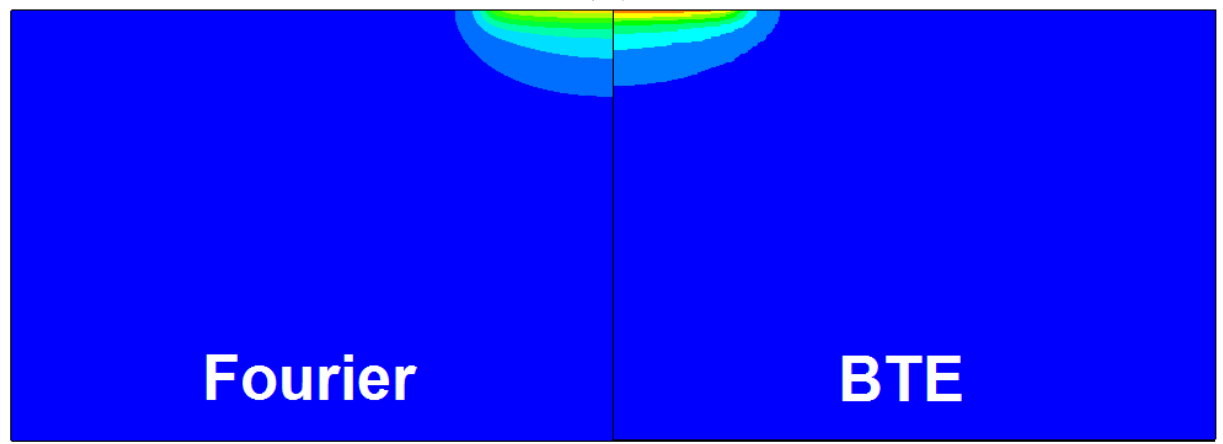

(c)

\section{Fourier}

\section{BTE}

(d)

Figure 8: Temperature distributions in the silicon substrate predicted by the Fourier law and the BTE at various instances of time: (a) $29.5 \mathrm{~ns}$, (b) $58.9 \mathrm{~ns}$, (c) $90.7 \mathrm{~ns}$, and (d) $118.8 \mathrm{~ns}$. 


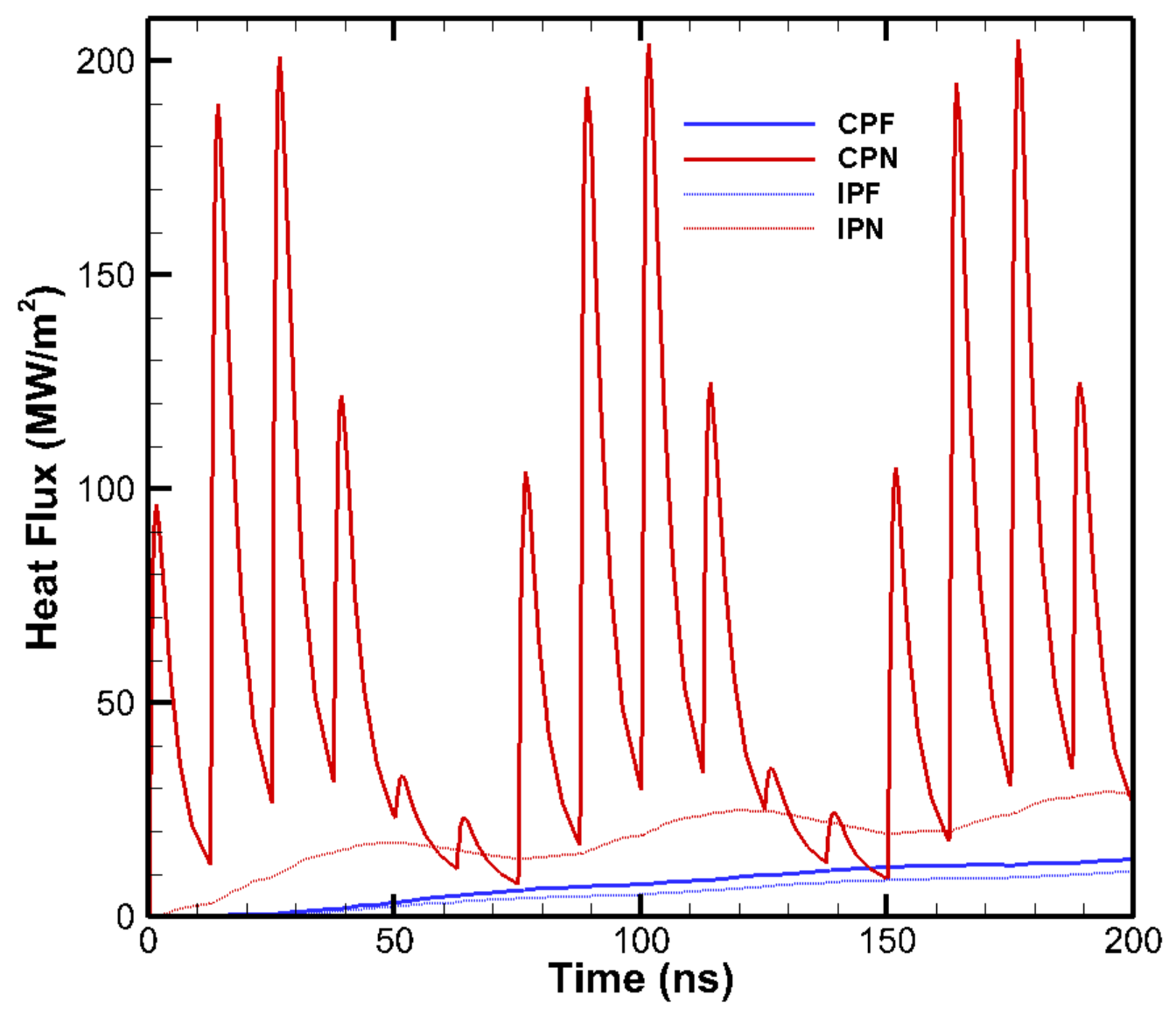

Figure 9: Temporal evolution of the heat flux at various probe locations for a spot size (radius) of $15 \mu \mathrm{m}$, and modulation frequency of $13.33 \mathrm{MHz}$. 


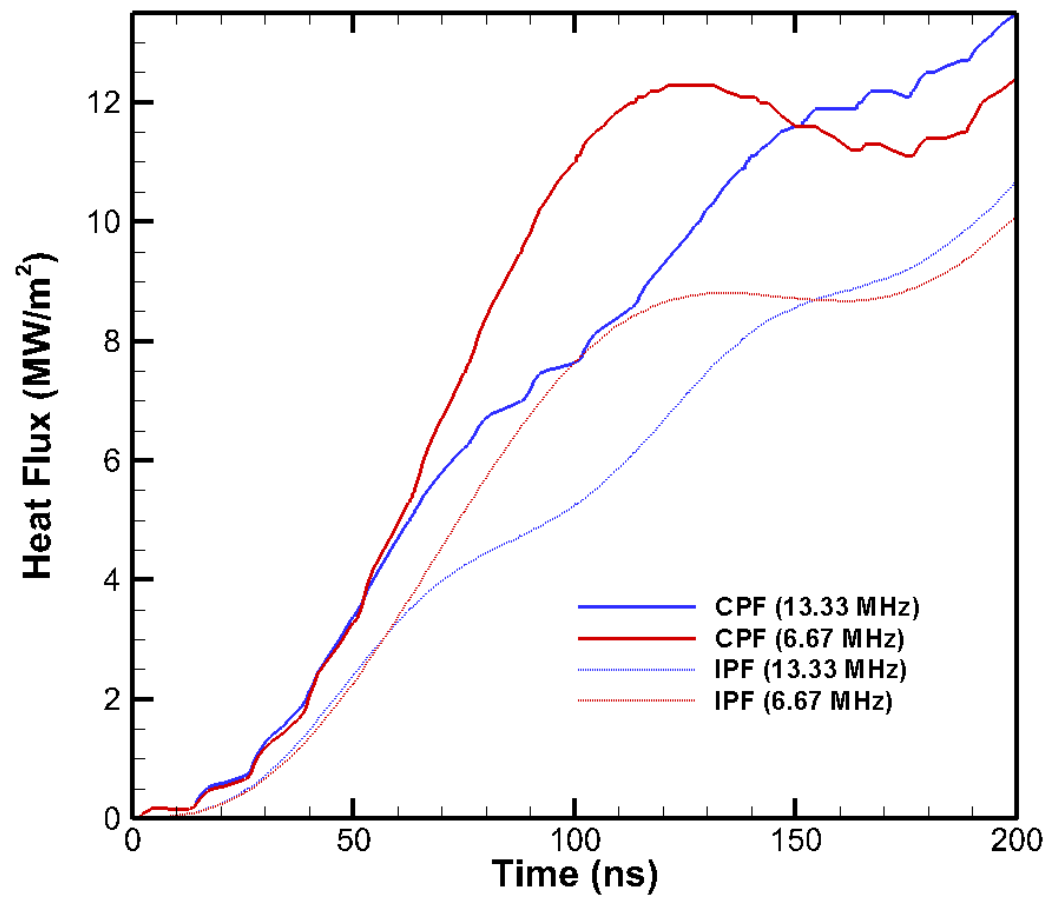

Figure 10: Temporal evolution of the effect of modulation frequency on normal heat fluxes at the four probe locations. A spot size (radius) of $15 \mu \mathrm{m}$ was used. 


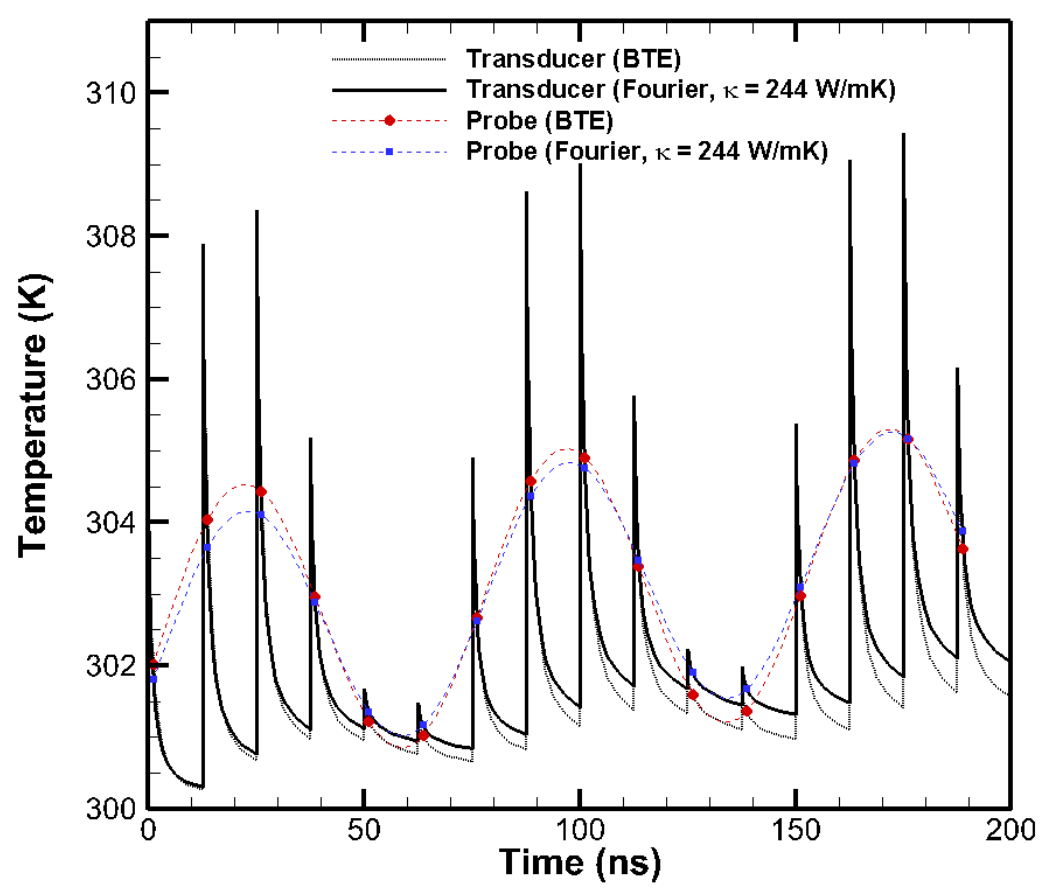

(a)

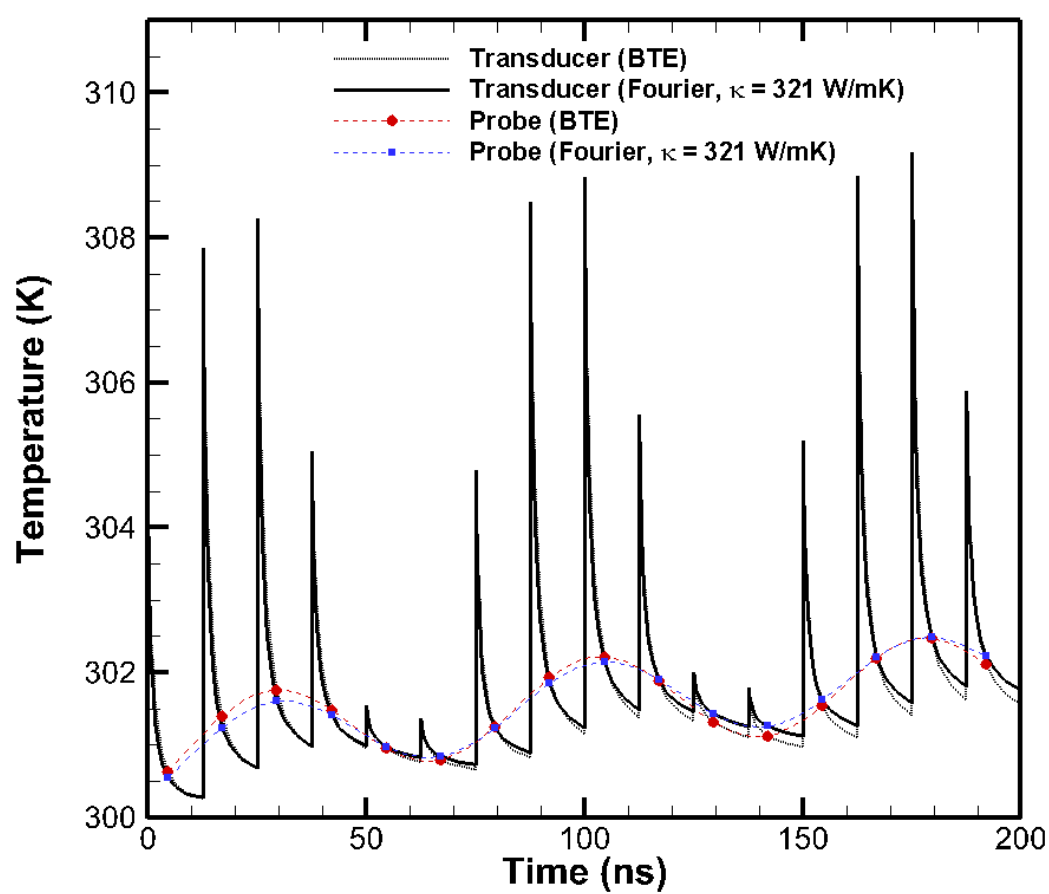

(b)

Figure 11: Fits obtained to BTE solutions using the Fourier law with two different probe laser time delays: (a) 1.14 ns after power pulse, (b) 4.49 ns after power pulse. 


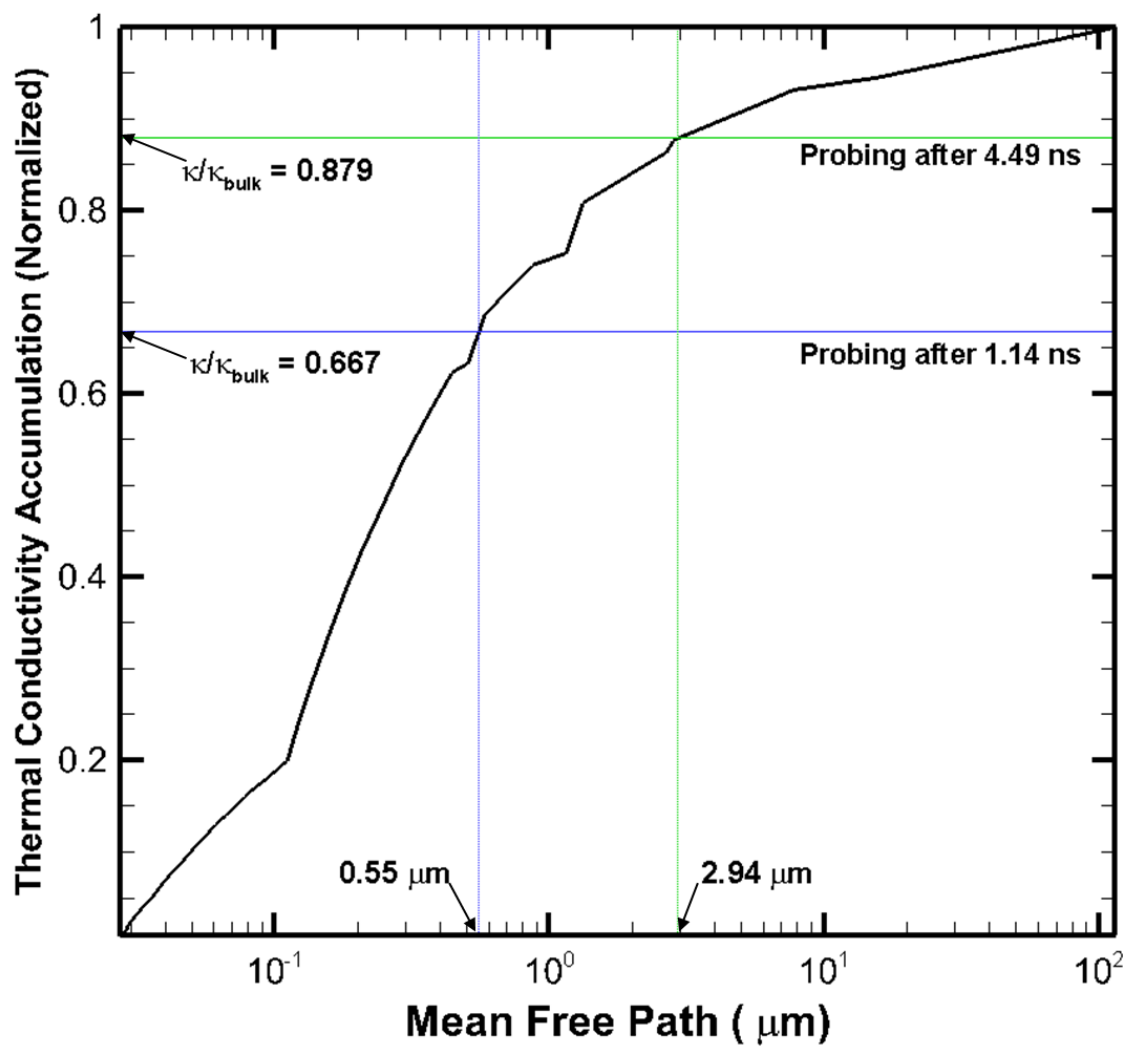

Figure 12: Thermal conductivity accumulation function along with the computed (from BTE) thermal conductivity for a spot size (radius) of $15 \mu \mathrm{m}$ and modulation frequency of 13.33 $\mathrm{MHz}$ with two different time delays for probing. 
Table 1: Location of virtual probes placed within the computational domain (see Fig. 5)

\begin{tabular}{|c|c|c|c|c|}
\hline $\begin{array}{c}\text { Cross-Plane Probe } \\
\text { locations }(\mu \mathrm{m}) ; \\
\text { distance from } \\
\text { top/symmetry }\end{array}$ & \multicolumn{5}{|c|}{$\begin{array}{c}\text { CPN: }(1.05,0.71) \\
\text { CPF: }(14.7,0.71)\end{array}$} \\
\hline $\begin{array}{c}\text { Spot size or radius } \\
(\mu \mathrm{m})\end{array}$ & 2.5 & 5 & 15 & 30 \\
\hline $\begin{array}{c}\text { In-Plane Probe } \\
\text { locations }(\mu \mathrm{m}) ; \\
\text { distance from } \\
\text { top/symmetry }\end{array}$ & IPN: $(4.93,2.7)$ & IPN: $(4.93,7.58)$ & IPN: $(4.93,15.95)$ & IPN: $(4.93,32.64)$ \\
& IPF: $(4.93,25.93)$ & IPF: $(4.93,30.00)$ & IPF: $(4.93,49.76)$ \\
& & & & \\
\hline
\end{tabular}


Table 2: Computed thermal conductivity using the BTE as a function of the pump laser modulation frequency and the spot size (radius). The bulk thermal conductivity is 366 $W / m / K$.

\begin{tabular}{|c|c|c|c|c|c|c|c|c|}
\hline \multirow[b]{4}{*}{$\begin{array}{l}\text { Spot size or } \\
\text { radius }(\mu \mathrm{m})\end{array}$} & \multicolumn{8}{|c|}{ Modulation Frequency (MHz) } \\
\hline & \multicolumn{4}{|c|}{6.67} & \multicolumn{4}{|c|}{13.33} \\
\hline & \multicolumn{2}{|c|}{$\begin{array}{c}\text { Probing after } \\
1.14 \mathrm{~ns}\end{array}$} & \multicolumn{2}{|c|}{$\begin{array}{c}\text { Probing after } \\
4.49 \mathrm{~ns}\end{array}$} & \multicolumn{2}{|c|}{$\begin{array}{c}\text { Probing after } \\
1.14 \mathrm{~ns}\end{array}$} & \multicolumn{2}{|c|}{$\begin{array}{c}\text { Probing afte } \\
4.49 \mathrm{~ns}\end{array}$} \\
\hline & $\kappa$ & $\kappa / \kappa_{\text {bulk }}$ & $\kappa$ & $\kappa / \kappa_{b u l k}$ & $\kappa$ & $\kappa / \kappa_{b u l k}$ & $\kappa$ & $\kappa / \kappa_{\text {bulk }}$ \\
\hline 30 & 229 & 0.62 & 304 & 0.83 & 229 & 0.62 & 304 & 0.83 \\
\hline 15 & 244 & 0.67 & 322 & 0.88 & 244 & 0.67 & 321 & 0.88 \\
\hline 5 & 237 & 0.65 & 311 & 0.85 & 238 & 0.65 & 311 & 0.85 \\
\hline 2.5 & 212 & 0.58 & 288 & 0.79 & 213 & 0.58 & 289 & 0.79 \\
\hline
\end{tabular}

